\title{
Gravitation, Quantum Mechanics and the Least Action Electromagnetic Equilibrium States
}

\section{Michaud A*}

Senior Researcher, Canada

\begin{abstract}
The century old challenge of fundamental physics has been to reconcile quantum mechanics (QM) that deals with submicroscopic interactions between elementary particles from the quantization perspective, with relativistic mechanics that deals with gravitation at the macroscopic level from the infinitesimally progressive perspective, mainly embodied by the theory of general relativity (GR). The ease with which infinitesimally progressive sequences of motion can be mathematically represented by means of an indefinite number of instantaneous momentary excited states of a postulated underlying neutral energy quantum vacuum field, which is the foundation of quantum field theory (QFT), has naturally privileged this quantization perspective in all past attempts at reconciling QM with gravitation. But, given that all scatterable elementary particles identifiable within atomic structures have an electric charge, and are thus electromagnetic in nature, this article explores the possibility of reconciling quantum mechanics with relativistic mechanics from the electromagnetic perspective, by means of reconciling the wave function with the least action electromagnetic resonance states into which elementary charged particles become captive within atomic and nuclear structures, and ultimately, with gravitation.
\end{abstract}

Keywords: Gravitation; Quantum Mechanics; Quantum Field Theory; Electromagnetism; Trispatial Geometry; Special Relativity; General Relativity

\section{Introduction}

This paper is the final instalment of a series of papers published in 2000, 2007, 2013, 2016 and 2017, describing the various aspects of an entirely new paradigm of fundamental physics, all of which are synthesized in a monograph published in 2017 by Scholars' Press [1] upon invitation by the editors.

The necessity of being able to refer to clear and concise explanations of each specific aspect of this new perspective resulted in the progressive publication of numerous separate papers, all of which having individually been peer-reviewed and accepted for publication, each of which relating in a self-contained manner a specific aspect of the new paradigm to the traditional paradigm.

The reader will certainly understand that the content of a 600 pages monograph synthesizing and completing the texts of about 20 separate papers certainly would be easier to explore via a simplified general overview, which the present paper is meant to provide.

\section{Maxwell Equations and Mutual Induction of Electric and Magnetic Fields}

The new paradigm is entirely grounded on an aspect of electromagnetic theory that has become obscured over time due to the generalizing perspective afforded by the use of the electromagnetic tensor, which represents both electric and magnetic fields as becoming a single entity that is the "electromagnetic field".

The downside of the otherwise usefulness of tensor treatment is that it conceptually obscures the fact that both $\mathrm{E}$ and B fields have different properties and represent different aspects of electromagnetic energy; in particular the fact that in physical reality, according to Maxwell's continuous wave theory, both fields can only mutually induce each other as revealed, among other characteristics, by the fact that the Poynting vector provides by structure the average value of the intensity of the product of the intensities of both time varying oscillating fields
[2] (p. 989). The Poynting vector indeed reveals that the time varying product of both fields in vacuum can only be constant:

$$
\mathrm{S}=\frac{\mathrm{EB}}{2 \mu_{0}}
$$

As clearly explained in traditional undergrad textbooks, such as "University Physics" by Sears, Zemansky and Young [3], or "Physics" by Halliday and Resnick [2], Faraday's law imposes that a time varying magnetic field acts as a source of electric field. This process is put in practice in the induction of electromotive force (emf) in inductances and transformers. Similarly, Ampere's law, which is used in charging capacitors and establish current in conductors, demonstrates that changing electric fields are a source of magnetic fields.

"Thus, when either field is changing with time, a field of the other kind is induced in adjacent regions of space. We are thus led naturally to consider the possibility of an electromagnetic disturbance, consisting of time-varying electric and magnetic fields, which can propagate through space from one region to another, even when there is no matter in the intervening region." ([3], p. 696).

Unfortunately such general and complete undergrad textbooks that were used in giving undergrad students a general knowledge of all aspects of fundamental physics, particularly in preparing them to smoothly transit from classical continuous processes to quantum and relativistic physics, progressively went out of fashion to be replaced by textbooks that barely skim over the classical concepts that were directly

${ }^{*}$ Corresponding author: Michaud A, Senior Researcher, Canada, Tel: 1418 6240608; E-mail: srp2@srpinc.org

Received September 22, 2017; Accepted November 02, 2017; Published November 06, 2017

Citation: Michaud A (2017) Gravitation, Quantum Mechanics and the Least Action Electromagnetic Equilibrium States. J Astrophys Aerospace Technol 5: 152. doi:10.4172/2329-6542.1000152

Copyright: () 2017 Michaud A. This is an open-access article distributed under the terms of the Creative Commons Attribution License, which permits unrestricted use, distribution, and reproduction in any medium, provided the original author and source are credited. 
extrapolated from the classical Equations, which were established by the major discoverers of the past from physical experiments that they actually carried out, and that constitute a pool of mutually converging conclusions about electromagnetic energy whose neglect can only lead to a lessening of our understanding of physical reality.

\section{Kinetic Energy and the Coulomb Law}

In traditional undergrad textbooks such as "Physics" by Halliday \& Resnick, the relation between the momentum related kinetic energy and the interaction between charges due to the Coulomb Force is established in the following manner.

From the electromagnetic Coulomb Equation applied to calculating the force between the charges of the electron and the proton in a hydrogen atom, taken as the traditional example, and the force calculated from Newton's second law for motion applied to the electron mass in motion ([2], p. 1192) and [4]:

$$
\mathrm{F}=\frac{\mathrm{e}^{2}}{4 \pi \varepsilon_{0} \mathrm{r}^{2}} \text { and } \mathrm{F}=\mathrm{ma}=\mathrm{m} \frac{\mathrm{v}^{2}}{\mathrm{r}}
$$

The following relation is drawn in Halliday \& Resnick:

$$
\frac{\mathrm{e}^{2}}{4 \pi \varepsilon_{0} \mathrm{r}^{2}}=\mathrm{m} \frac{\mathrm{v}^{2}}{\mathrm{r}}
$$

which allows calculating the momentum related kinetic energy of the electron from Newton's kinetic energy Equation (Equation 7-19) [2]:

$$
\mathrm{K}=\frac{1}{2} \mathrm{mv}^{2}=\frac{\mathrm{e}^{2}}{8 \pi \varepsilon_{0} \mathrm{r}}
$$

which is how the kinetic energy sustaining the momentum of charged particles is related to the Coulomb force as a function of the distance separating pairs of charges, because the only variable in the Coulomb Equation is " $r$ ", which is the mean distance separating the electron stabilized in the ground state orbital and the proton in the hydrogen atom, which causes any amount of momentum kinetic energy that a charge may possess to be dependent solely on the distances separating it from other charges. Consequently, the closer charges come to each other, the more momentum related kinetic energy they will be induced with, given that the force is acting as a function of the "inverse" square of the distance.

The issue of potential energy is addressed further on in the section dealing with the momentum, the Lagrangian and the Hamiltonian and is completely analyzed in correlation with energy conservation in closed systems [5].

But there is more! In 1903, Walter Kaufmann was the first experimentalist to relate the gamma factor to energy induction during experiments carried out with electrons moving at relativistic velocities in a bubble chamber by accelerating and deflecting them with a combination of electric and magnetic fields [6], by demonstrating that their transverse mass varied with velocity in conformity with the relativistic Equation [7]; experiments that he was carrying out in collaboration with theoreticians Max Abraham [8] and Woldemar Voigt, who is the physicist who initially conceived of the gamma factor [9], better known as the Lorentz factor.

So it seems that the gamma factor was initially experimentally related strictly to energy and mass induction with velocity, an experimental result with which Henri Poincare was in agreement:

"Abraham's calculation and Kaufmann's experiments then showed that mechanical mass proper is null and that the mass of electrons is exclusively of electro-dynamic origin. This forces us to change the definition of mass; we can no more distinguish mechanical mass from electrodynamic mass, because then the first would disappear; there is no other mass than electrodynamic inertia; and in this case, mass can no longer be constant, it increases with velocity; and which is more, it depends on the direction, and a body animated with a notable velocity will not oppose the same inertia to forces tending to deflect its trajectory, and to those who tend to accelerate or slow its progress." Henri Poincare ([10], p. 137).

It is a historical fact that these physicists who worked closely with the discoverer of the method never accepted the interpretation that the gamma factor could be axiomatically related later to time dilation and length contraction of bodies with velocity.

This means that not only is the momentum related kinetic energy of the electron induced by the Coulomb force, but the energy that serves to increase the mass of a moving electron is also induced by at least one of the ambient electric and magnetic fields, presumably in context the electric field, given its relation to the Coulomb force, which means that the total complement of energy induced in a charged particle by the electric field related Coulomb force can be calculated with the following Equation directly drawn from Equation (3):

$$
\mathrm{K}_{\text {Total }}=\mathrm{mv}^{2}=\frac{\mathrm{e}^{2}}{4 \pi \varepsilon_{0} \mathrm{r}}
$$

which is the total amount of induced energy that Leibnitz already considered in Newton's era as being the real effect of application of a force ([2], p. 222).

\section{Special Relativity and the Gamma Factor}

As previously mentioned, the gamma factor on its part was first established by Woldemar Voigt in 1887 [9], for whom there are on record epistolary contacts with Larmor, Lorentz and Poincare, who also are credited with developing the method. This method is clearly laid out in a very well done paper published in 2003 by Richard E. Haskell [11]. Indeed, it is clear from this description, that the gamma factor was established strictly from formal geometric and trigonometric considerations unrelated to electromagnetism.

On page 10 of reference [11], the first postulate of Special Relativity is summarized as resolving to the following statement "Absolute uniform motion cannot be detected by any means." and the second postulate is formulated as "Light is propagated in empty space with a velocity $c$ which is independent of the motion of the source".

It must be noted here that these postulates are presented as being axiomatic in nature, since they are not presented as deriving from underlying experimentally established physical causes.

It is useful also to note here that these postulates were proposed in this axiomatic manner by Einstein in 1905 without any mention of the fact that the constant velocity of light in vacuum and its known exact velocity of $c=299792458 \mathrm{~m} / \mathrm{s}$ had been established 40 years earlier by Maxwell from second partial derivatives of Gauss and Ampere Equations, that linked both electric and magnetic fields as mutually inducing each other in a manner that could only result in this stable velocity of electromagnetic energy in vacuum.

It must thus be realized that the totally conclusive experimental verifications of the speed of electromagnetic energy in vacuum by a variety of means over the course of the past century indeed first and foremost validate Maxwell's calculations, which were arrived at not axiomatically, but were derived from Equations experimentally established by Gauss and Ampere. Indeed, the constancy of the speed of light is so well established experimentally that in 1983, the SI meter 
was redefined as being the experimentally confirmed and fixed distance covered by light in 1 second divided by 299792458 .

So, this experimental confirmation of the absolute uniform motion of light in vacuum renders invalid the first postulate as formulated, and the second postulate turns out not to be axiomatic, but a direct conclusion derived from electromagnetism and subsequent experimental confirmation.

To establish the constancy of the speed of light as the foundation of the SR theory, the traditional procedure makes use of the famous relation between two different reference frames moving inertially at different constant velocities, each harboring an observer immobile in his own reference frame, both having the task of measuring the speed of a light beam to be the same for both observers.

As described on page 10 again, the traditional set up involves that one of the inertial reference frames be a train moving at a fixed velocity, and that if a light signal was emitted from the back of the train to the front, then both an observer on the train and an observer on the ground should be able to measure the velocity of the light signal as being "c".

Then is exposed the logically well grounded geometric construct that allows associating with Equation (5) of reference [11] a squared velocities ratio $" \mathrm{v}^{2} / \mathrm{c}^{2 "}$ to the "sin" component in the well known trigonometric function " $\sin ^{2} \theta+\cos ^{2} \theta=1$ ", to then establish a precursor to the gamma factor as being related to time (but also axiomatically to the concept of time dilation) with Equation (6) of reference [11]. It is quite interesting to note at this point that this particular trigonometric function can also be used to describe the mutual induction of the electric and magnetic fields of localized quanta such as electromagnetic photons [12], as we will see further on.

It is to be noted also that "c" is axiomatically introduced in this relation without reference to its prior establishment from experimentally defined electromagnetic Equations by Maxwell. The same procedure is then used to relate the same squared ratio of velocities to the "cos" component of the same trigonometric function to relate the length of the train (thus length contraction) to this other precursor of the gamma factor as Equation (8) of reference [11].

The gamma factor is then formally established with Equation (14) of reference [11] as being related to time dilation and length contraction of moving bodies. The remainder of Part II and Part III describe the Lorentz transformation and relativistic dynamics from the Special Relativity perspective.

\section{Disconnect between the distance-dependence of energy induction and the concept of SR length-contraction}

It is at this point that the distance-dependence of kinetic energy induction in charged particles by the Coulomb force as established with Equation (47-19) of reference [2] previously reproduced as Equation (4) must be brought to mind again, because there is a clear disconnect between this property of the Coulomb force which is in permanent action between charged particles and the concept of length contraction as applied in special relativity to moving macroscopic bodies.

To correctly put this issue in perspective, it is important to become aware of the physical distances separating electronic escorts from nuclei within atoms. If for example a hydrogen atom was upsized so that the proton became as large as the Sun, then the electron would stabilize as far as Neptune's orbit, which would make the whole atom as large as the solar system! This means that all proportions considered, the distances separating electronic escorts from nuclei within atoms are relatively astronomical with respect to the sizes of elementary particles.

Given that all macroscopic bodies are made of such practically "empty" structures, the very concept of "length" becomes meaningless with respect to their internal composition, and what would be involved when the possible "length contraction" of a macroscopic body is considered, would really be a "distance contraction" between the electronic escorts and the nuclei of the constituting atoms, which is the only way that the physical length of a rigid macroscopic body can be diminished without deformation.

This being said, such distance contraction would apply by structure not only to the length of macroscopic bodies, but also to their other dimensions, which are their width and thickness, and such shortening of the distances between the charged electrons of the electronic escorts and their charged atomic nuclei within bodies subjected to "length contraction" would then involve by structure a corresponding energy increase within the mass of the body due to the now increased intensity of the Coulomb force at these shorter distances between the charges.

However, no such increase in energy is even considered in SR in relation to "length contraction" of moving macroscopic bodies, which means that despite the general assumption that SR is electromagnetism compliant, it really is not, because the Coulomb law is at the heart of Gauss's Equation for the electric field, and is in fact Maxwell's first Equation, from which the Coulomb Equation (2) can easily be derived [13],

Another major issue can also be raised with regard to the relation between the gamma factor axiomatically established from strictly geometric and trigonometric considerations relating it to time dilation and length contraction, and its use to conclude that Maxwell's Equations and Lorentz's force Equation can be derived from SR as described in Part IV of reference [11]. Since the gamma factor seems to never have been derived from an electromagnetic Equation, such an interconnection of electromagnetism to time dilation and length contraction is at best axiomatic.

Indeed, despite a long and fruitless search in formal literature for such a derivation, evidence seems to reveal that a first time derivation of the gamma factor from an electromagnetic Equation was effectively carried out and published only in 2013, as Equation (66) [14], derived from Equation (51) of the same reference, itself a conversion from strictly electromagnetic Equation (34) of the same reference, and from which all relativistic Equations can be derived $[1,14]$.

Equation (34) from reference [14] is indeed derived in direct line from the Biot-Savart Equation via a seamless derivation by Paul Marmet directly relating the relativistic mass increase of a moving electron to a simultaneous increase of its magnetic field with velocity $[14,15]$.

And even if a prior derivation of the gamma factor from an electromagnetic Equation had been carried out while escaping the attention of this author, the outcome would be the same, because it can effectively be verified that from the electromagnetic perspective the "gamma factor" derived [14] has nothing to do with time dilation nor length contraction, but is strictly related with charged particles momentum related kinetic energy increase with velocity and proximity between charged particles according to the Coulomb law, in accordance with Equation (5), and in conformity with the conclusions of Voigt, Abraham and Poincare with regard to Kaufmann's experiments $[6,8,9,10]$.

So, whatever dimensions may be associated to the varying ratio of the gamma factor, $\mathrm{m} / \mathrm{s}$, joules or $\mathrm{kg}$, these dimensions always simplify completely out of whatever calculation the gamma factor may be 
involve in, which means that the Lorentz factor is only a special case of an intrinsically dimensionless mathematical function that can be used in a general manner to introduce the denominator of the ratio as the asymptotic limit of a growth curve obeying the power of the ratio, in the present case, the squared ratio and the asymptotic limit derived from an electromagnetic equation.

Consequently, there seems to be ample reasons to question the compliance of SR with Maxwell Equations, and there is also reason to question the reality of time dilation and length contraction axiomatically related to the gamma factor as established from strictly geometric and trigonometric considerations when put in perspective with respect to the direct derivation of the same gamma factor from an electromagnetic Equation that relates it strictly to the variation of energy induced by the Coulomb force as a function of the distances separating charged particles.

It goes without saying that such questioning of the reality of time dilation and length contraction axiomatically established as a foundation of SR also brings in question the space-time curvature of the General Relativity theory and all of the axiomatic conclusions that the theory leads to. It must be emphasized here that Einstein himself had become convinced toward the end of his life that gravitation follows the patterns of electromagnetism ([16], p. 391), which means that he also had come to doubt the validity of his own brainchildren SR and GR theories.

These considerations are at the heart of the development of the present possible alternate solution entirely derived from the converging set of electromagnetic Equations experimentally established by Coulomb, Ampere, Gauss, Faraday, Maxwell, Lorentz, Biot and Savart, without any axiomatic assumptions.

One of its main objectives was to attempt addressing one of the major hurdles of fundamental physics which is summarized in this remark by Feynman mentioned during his famous "Feynman Lectures on Physics" [17]:

"There are difficulties associated with the ideas of Maxwell's theory which are not solved by and not directly associated with quantum mechanics... when electromagnetism is joined to quantum mechanics, the difficulties remain"

This author is convinced that by clearly defining the self-sustaining mutual induction of the electric and magnetic fields of the energy quanta making up localized electromagnetic elementary particles such as the electromagnetic photon and the electron, this hurdle will be resolved.

Permanent localization of the electron when in motion is maintained in this new paradigm by allowing the definition of a clear resonance trajectory of the moving electron within the volume defined by the wave function.

\section{Establishment of the fundamental equations from physically collected data}

One of the major difficulties in fundamental physics is the very power of mathematics as a descriptive language. If care is not taken to avoid as much as possible axiomatic postulates, an indefinite number of theories can be elaborated with full mathematical support that can always become entirely self-consistent with respect to the set of premises from which each theory is grounded. But the very self-consistency of all well thought out theories is so appealing to our rational minds that it renders very difficult the requestioning of the grounding foundations of such beautiful and intellectually satisfying structures and consequently the identification of possibly inappropriate axiomatic assumption.
Given however that there exists only one physical reality, it would seem that only one explanation would correctly address each of its aspects and that the related theories are likely to more appropriately describe it if axiomatic assumptions are avoided as much as possible in grounding their elaboration.

Before drawing any conclusion about physical reality, experimental data about this physical reality must obviously be first collected, that then allows extrapolating hypotheses that would explain this data. Although it is relatively easy to confirm the validity of this data by repeatedly obtaining the same results from various experimental means, the same cannot be said of the theories established from the interpretation of this data.

For example, the unit charge of the electron has been conclusively measured out of any possible doubt over the course of the past century, to have an absolutely invariant value. Therefore, this characteristic of the electron is considered an objectively valid component of any set of premises to be used in drawing conclusions about its nature. However, the conclusion as to whether the electron remains localized while moving, as dealt with from the relativistic mechanics perspective, or whether its "substance" spreads out when moving according to the wave function, as dealt with from the quantum mechanics perspective depends entirely on the other elements in the set of premises on which each theory is grounded.

Other conclusively confirmed characteristics of the electron are the invariance of its rest mass, the fact that it always behaves pointlike during scattering encounters and that it presumably possesses an indefinite life span unless physically converted to energy during very specific accidental interaction events with other elementary particles, which allows considering it as being "stable".

Since all matter in existence is made of massive atoms, gravitation logically must be emergent from the properties of these atoms. In turn, all atoms being ultimately made of a very restricted set of charged and massive elementary particles locked in mutual interaction, this logically implies that the properties of atoms must ultimately be emergent from the properties of these elementary subcomponents.

This ultimate set of stable, charged and massive elementary particles making up the internal structure of all atoms is very limited, and their existence has been confirmed out of any doubt by means of non-destructive scattering. There are only 3 of them, that is, the electron, that establishes the electronic escorts of atoms about their nuclei, which determines atomic volumes; and the up and down quarks that were found to be the ultimate charged and massive elementary subcomponents of all nucleons inside atomic nuclei, that determine their volume, and that were first detected by deep non-destructive scattering during the first years of operation of the Stanford Linear accelerator (SLAC), from 1966 to 1968 [18].

These three charged particles are considered elementary because no scattering experiment ever revealed the existence an unbreachable limit at some distance from their center that would have related them to a measurable volume, as was the case for protons and neutrons, when scattered against with insufficient energy, which was the unmistakable telltale that nucleons are not elementary and have an internal structure involving smaller particles, that turned out to be the up and down quarks just mentioned, observed to be interacting in triads of both types, that is, uud for the proton and udd for the neutron.

These three particles being elementary, their masses must then logically be made of some undifferentiated substance, which was identified in the case of the electron as being electromagnetic energy, 
given its electric and magnetic properties, and the same conclusion can be reached by similarity for the up and down quarks for the same reason.

We also know that electromagnetic energy is intimately linked to momentum related kinetic energy, because we have undisputable evidence that the exact amounts of kinetic energy accumulated by electrons accelerating between the electrodes of a Coolidge tube, for example, due to the Coulomb force in action between the accelerating negatively charged electrons and the positively ionized atoms of the anode, are liberated as electromagnetic X-ray photons when they are suddenly stopped in their translational motion, when momentarily captured by the positively ionized atoms of the anode (or anti-cathode).

We thus observe that from the electromagnetism perspective, the Coulomb force, that we know to be in continuous action between all charged particles in existence, belongs to the deepest layer of physical reality with regard to the induction of kinetic energy in accelerating charged elementary particles. Therefore, this force can be identified as the ultimate cause the very existence of kinetic energy at the submicroscopic level. We also know from the experimental evidence provided by the Coolidge tube operation, that as it escapes as bremmsstrahlung photons, this induced kinetic energy displays the same electromagnetic properties already associated with the restricted set of the three charged and massive electromagnetic elementary particles that are the only building blocks of all atoms in existence.

\section{Procedure}

This article will first put in perspective an aspect of electromagnetic energy yet unclarified in all currently useful physics theories, which is the fact that none of these theories provides a mechanical description of the self-sustaining mutual induction of the electric and magnetic fields of the energy making up the rest mass of elementary particles, that would be consistent with their point-like localization observed during their mutual scattering encounters, which is the mutual induction of electric and magnetic fields that justifies the very existence of electromagnetic energy in Maxwell's theory.

A possible description of this mutual induction in the frame of an expanded orthogonal space geometry will be proposed that brings to light a set of properties that allows mechanically explaining electronic and nucleonic orbitals stability in atoms.

The function of the Coulomb force in electromagnetic energy induction will be analyzed, and a related analysis will follow of the disconnect that this new perspective reveals between the current energy conservation principle based concepts of momentum/Lagrangian/ Hamiltonian and the currently unaccounted for motion hindered adiabatic kinetic energy permanently induced in these three charged elementary particles captive in various resonance states within atomic and nucleon structures.

The relation between these least action electromagnetic resonance states and the wave function as well as with gravitation will finally be put in perspective, as well as the possibility brought to light that the methods of quantum mechanics could be directly applied to nucleons inner structures.

\section{The inner electromagnetic structure of electrons}

One characteristic of the electron yet to be mentioned, was suspected by Louis de Broglie in the 1920's, and was experimentally confirmed in the 1930's. It is the fact that the very substance of which its invariant rest mass is made is actually electromagnetic energy, as established by the repeatedly confirmed fact, initially discovered by Blackett and Occhialini [19], that massless electromagnetic photons of $1.022 \mathrm{MeV}$ or more can be destabilized into converting to massive electron-positron pairs and that the masses of a pair of electron and positron metastabilizing into positronium configuration will reconvert back to massless electromagnetic photon state as the final inward spiralling stage of the positronium decay process, which was also initially confirmed by Blackett and Occhialini in the same period. Confirming evidence of the electromagnetic nature of the mass of these two particles is of course that it they are electrically charged and are conclusively known to possess a magnetic moment.

These intrinsic electromagnetic properties of the energy constituting the rest mass of the electron are however not clearly integrated into its wave function representations, nor are they integrated into the concept of localized mass addressed by relativistic mechanics.

\section{No description of the electron internal electromagnetic structure in classical and relativistic mechanics}

Relativistic mechanics treats all massive bodies, including electrons, as if they had no internal structure, which sometimes leads to results that are difficult to relate to otherwise well established laws.

For example, in traditional classical and relativistic mechanics, this difficulty is made particularly obvious with respect to the rotating motion of massive bodies, whose angular momentum is deemed to be conservative, which is a conclusion that disregards the fact that in physical reality, all macroscopic rotating masses can only be made of the sum of the masses of a number of captive elementary massive particles translating on circular orbits about the body's axis of rotation, all of which are individually subject to the $2^{\text {nd }}$ principle of thermodynamics, that mandates that the constant change in direction imposed on these massive sub-components de facto involves an expenditure of energy as work, which comes in contradiction with defining rotation as being conservative, given that it is impossible, according to the $2^{\text {nd }}$ principle of thermodynamics, that the state of motion of massive bodies such as these massive elementary particles could constantly change in this manner without an expenditure of energy.

Could this be related to the unexplained observed rotation slowing down of all bodies left to rotate in deep vacuum for extended periods of time after having been set in rotation from an initial impulse, such as both Pioneer 10 and 11 spacecrafts ([20], p. 23)? Or the ball bearing of J.C. Keith's experiment in 1963 [21], that was made to rotate frictionlessly at high velocities in deep vacuum while suspended in magnetic fields? Or the ball bearing in a similar confirming experiment conducted by J.K. Fremerey in 1973 [22]? Or even of individual electrons being made to translate on perfectly circular orbits in the Betatron during J.P. Blewett's experiments in 1946 ([23], p. 87)?

Unfortunately, the case of the still unexplained electron translational slowdown observed by Blewett was not investigated further and was neglectfully left hanging without an answer to this day, after the Betatron was decommissioned before he could investigate further. Various reassuring rationalizations were applied to all other slowdown cases that did not force reconsideration of the assumed "conservative" nature of rotating motion.

It can nevertheless be observed that all elementary massive particles captive inside macroscopic rotating bodies are translating on perfectly circular macroscopic scale orbits identical to that of the isolated electrons observed by Blewett in the Betatron, which is the only accelerator type that allows perfectly circular orbits for isolated electrons. It would be highly interesting indeed if the still to be investigated unexplained slowdown of the Betatron electrons was finally studied in depth and correlated with the slowdown observed for 
rotating bodies, taking into consideration the identical circular orbits that elementary charged and massive particles are forced into within macroscopic bodies.

Interestingly, it wouldn't come to anybody's mind to consider the solar system as being a massive body devoid of an internal structure, because we can directly observe that it is a stabilized system of smaller bodies and that its total mass is the sum of the masses of these individual smaller bodies. This is nevertheless what is being done when not assuming an internal structure to macroscopic massive bodies, because it is not the macroscopic bodies themselves that are massive, but the individual submicroscopic elementary massive particles whose sum of individual masses add up to make up the total mass of any macroscopic massive body.

Not assuming an internal structure for macroscopic masses, classical and relativistic mechanics do not assume any internal structure either for the rest mass of elementary electromagnetic massive particles such as the electron, which led to the default unexpressed assumption that the electron has such a "volume", simply by finding no fault with the concept of magnetic spin as corresponding to an angular momentum, since the very concept of "rotation" mandates the presence of such a volume, which disregards the fact that no scattering experiment ever detected any unbreachable limit at some distance from the point-like center of electrons that would have related them to such a measurable volume, as was the case for protons and neutrons.

Indeed, the only logically possible cyclic process that could animate a volumeless object as the electron, that behaves point-like during all scattering encounters, seems to be some sort of reciprocating internal motion, a hypothesis that will be supported by the manner in which the self-sustaining internal mutual induction of the electric and magnetic fields of the electromagnetic energy quantum making up its invariant rest mass can be represented in an expanded orthogonal space geometry, as will be shown further on.

\section{No description of the electron internal electromagnetic structure in quantum mechanics}

Quantum mechanics on its part currently offers three different descriptions of the electron in motion, which comprises the energy making up its rest mass plus its momentum energy, but does not offer separate representations of these two quantities.

The first representation stems from Schrödinger's wave function description that he established to represent the resonance states that de Broglie had previously concluded that electrons had to be captive into when stabilized about atomic nuclei [24]. Stated in general terms, this representation describes the electron energy as being spread out within the volumes definable with the wave function.

The second representation was simultaneously and independently developed by Heisenberg, which spreads the energy of the electron within the volume otherwise defined by the wave function according to statistical probabilities of density presence of the energy of which the electron is made, which allows for example defining the area of greatest probable density of the electron "substance" in the hydrogen ground state as corresponding to the ground state orbit of the classical Bohr atom.

The third representation is the path integral subsequently developed by Feynman, which replaces the theoretical least action trajectory of an electron in motion with the infinity of possible trajectories that the electron could possibly run within the volume defined by the wave function.
It can also be observed that besides not representing separately the carrying energy of the electron from its invariant rest mass, none of these current QM representations offers a description of the selfsustaining mutual electric and magnetic induction of the energy quantum making up its invariant rest mass.

We will see further on how a possible fourth representation, that makes use of such a description may allow describing the actual resonance trajectory of a permanently localized electron within the volume defined by the wave function in the ground state of the hydrogen atom, thus proposing a possible general method to allow resonance trajectories representation of localized charged elementary particles within all atomic and nuclear orbitals within the limiting volumes definable by the wave function.

\section{No description of elementary particles internal electromag- netic structure in quantum field theory}

The more general quantum field theory (QFT) assumes that elementary electromagnetic particles such as the electron emerge as "local excited states" of an underlying neutral quantum energy field, thus introducing the concept of energy quantization that gave rise to quantum electrodynamics (QED), which allows the description of interactions between elementary particles as quantized "virtual exchange photons".

But it can be noted that QFT, although grounded on electromagnetism, provides no description either of the actual internal mutual induction process of both electric and magnetic fields of these individual excited states.

\section{No description of the electron internal electromagnetic structure in electromagnetism}

Surprisingly, even in electromagnetism as currently formulated, although the very foundation of Maxwell's theory mandates that the electric and magnetic fields of free moving electromagnetic energy need to cyclically induce each other for electromagnetic energy to even exist, it has not proved possible to this day to coherently represent this cyclic self-sustaining mutual induction within localized electromagnetic photons, nor within localized elementary electromagnetic particles such as the electron.

Indeed, it was the observation that a mechanical description of this internal mutual electric and magnetic fields induction was lacking in all of these generally useful theories about matter and energy that brought to light the possibility that resolving this particular issue might clarify some aspects of electromagnetic energy that could be key to completely reconcile these theories with each other and with objective reality.

As quantum mechanics was being established in the 1920's, it was already obvious of course that electromagnetism needed to be correlated with the newly developed wave function due to the prior establishment by H.A. Lorentz of the ground-breaking first ever Equation of electromagnetic mechanics $\mathbf{F}=\mathrm{q}(\mathbf{E}+\mathbf{v} \times \mathbf{B})$, that allowed controlling the motion of electrons on precise trajectories by varying the relative densities of ambient electric and magnetic fields, equal densities providing motion in straight line of the charged particle.

So, very early after the advent of Schrödinger's wave function and Heisenberg's statistical method, this disconnect between QM and electromagnetism eventually gave rise to the development of QFT, which led to the introduction of the quantization perspective.

Louis de Broglie on the other hand, remained intimately convinced that electromagnetic photons and electrons had to remain permanently 
localized and follow precise trajectories when in motion. He then undertook to establish the internal electromagnetic structure for the localized electromagnetic photon [25-28], but his decade long attempt in the 1930's not succeeding in correlating this internal mutual induction in harmony with the wave function, led him to conclude that it was impossible to exactly represent elementary particles in the frame of the 4 dimensional space-time geometry, also adding that this should eventually become possible by escaping from this presumably too restrictive spacetime frame ([29], p. 273).

Even today, we know that light can be polarized, but we do not have a mechanical description explaining why electromagnetic energy can be polarized.

Even if we know that electromagnetic energy involves a process of mutual electric and magnetic fields induction, we still do not have a mechanical description explaining why the electromagnetic energy quantum that makes up the rest mass of an electromagnetic elementary particle such as the electron can remain localized while its internal electric and magnetic fields mutually induce each other in the selfsustaining manner that we can observe.

We know that only three stable, charged and massive electromagnetic elementary particles are the only building blocks of all atoms in the universe (electron, up quark and down quark), but we do not know yet why their electromagnetic energy quanta, made of selfsustaining and mutually inducing electric and magnetic fields, remain localized to display the point-like behavior that we can observe when they scatter against each other.

Since gravity is apparently related to mass, it must by structure be related to the only three existing stable self-sustaining electromagnetic elementary particles that display mass and that obviously are the only and ultimate massive building blocks of all existing atoms in the universe.

\section{Establishing the internal structure of electromagnetic photons}

Following de Broglie's intuition that $4 \mathrm{D}$ spacetime geometry seemed too restrictive to establish this mechanics, a new expanded space geometry was eventually developed and proposed in 2000 [30], that relates the triply orthogonal relation of electromagnetic energy revealed by plane wave treatment with the orthogonality of space itself, that effectively allows mechanical representation as Equation (6) of the internal mutual induction of the electric and magnetic aspects of the electromagnetic energy of a localized photon as hypothesized by de Broglie, in conformity with Maxwell's Equations, in a manner that can explain polarization [12]. This new expanded space geometry is put in perspective with respect to other more familiar multidimensional attempts at resolving the remaining issues in fundamental physics [31], and is completely described [12].

Summarized in a few words, this expanded space geometry stems directly from the well-known triple orthogonal vectorial relation of electromagnetism that maps any point of the wavefront of Maxwell's continuous electromagnetic wave as a cross product of the magnetic field vs. the electric field, both perpendicular to the direction of motion of any point of the wavefront in plane wave treatment. The new geometry results from metaphorically "exploding" each of the three related mutually orthogonal ijk vectors into full blown $3 \mathrm{D}$ vectorial spaces mutually orthogonal to each other, while the central junction point of all unit vectors of such vector complexes remains located at the center of each localized electromagnetic quantum.
For example, the following trispatial LC Equation (6) for the selfpropelling localized electromagnetic photon clearly describes in this expanded space geometry, how half of its energy transversally oscillates between a state of two electric components, which is key to explaining polarization in conformity with de Broglie's hypothesis, and a single magnetic state, that insures permanent localization of the quantum in complete conformity with Maxwell's Equations; while the other half remains unidirectional, and perpendicular to the transversally oscillating half, sustaining the momentum related equilibrium speed of light of the whole quantum in vacuum, without any need for an underlying ether, while its default equal electric and magnetic fields densities ensure self-guidance in straight line when no external electromagnetic fields modifies this default equal densities equilibrium ratio in a manner that would deflect its trajectory [12]:

$$
E \vec{I} \vec{i}=\left(\frac{h c}{2 \lambda}\right)_{X} \vec{I} \vec{i}+\left[\begin{array}{c}
2\left(\frac{e^{2}}{4 C}\right)_{Y}(\vec{J} \vec{j}, \vec{J} \overleftarrow{j}) \cos ^{2}(\omega t) \\
+\left(\frac{L i^{2}}{2}\right)_{Z} \stackrel{\leftrightarrow}{K} \sin ^{2}(\omega t)
\end{array}\right]
$$

where

$$
\mathrm{C}=2 \varepsilon_{0} \alpha \lambda \quad \mathrm{L}=\frac{\mu_{0} \alpha \lambda}{8 \pi^{2}} \mathrm{i}=\frac{2 \pi \mathrm{ec}}{\alpha \lambda} \omega=\frac{2 \pi \mathrm{c}}{\alpha \lambda}
$$

Establishing the internal electromagnetic structure of the carrying energy of massive elementary particles

Regarding a possible representation of the internal electromagnetic structure of the energy of the rest mass of the electron with respect to relativistic mechanics, a groundbreaking breakthrough was made by Paul Marmet in 2003, when he succeeded in deriving from the BiotSavart Equation a relation that directly relates the relativistic mass increase of an accelerating electron to the simultaneous increase of its magnetic field [15], which led to observe that the magnetic field of the electron at rest corresponds to precisely half of its invariant rest mass, which in turn led to conclude that the other half of its invariant rest mass had to correspond to its electric field.

This means that by structure, the electron measurable velocity related relativistic magnetic mass increment can only involve its carrying energy as being distinct from the energy making up its invariant rest mass energy quantum, in a manner that causes it to acquire the same magnetic mass characteristics as the invariant rest mass of the electron [32], that is, a property of omnidirectional inertia in normal space corresponding to the established concept of electromagnetic mass.

Indeed, since the traditional momentum related kinetic energy that propels the electron can only be vectorially unidirectional by structure as it translationally propels the electron, and that in accordance with Maxwell Equations' fundamental vectorial requirement that a magnetic field be by structure oriented perpendicularly to the direction of motion of electromagnetic energy, then the magnetic field of the velocity related mass increment contributed by the energy in excess of the electron invariant rest mass, can only be part of a transversally oriented energy component different from the translationally oriented momentum energy component of the carrying energy, which thus exists by structure separately from the particle invariant rest mass electromagnetic energy quantum:

$\mathrm{E}_{\text {total carrying energy }}=\mathrm{E}_{\text {translational }}+\mathrm{E}$

Given that a magnetic field cannot be dissociated from an electric counterpart in Maxwell's theory, and that both aspects mandatorily 
have to mutually induce each other for electromagnetic energy to even exist, then the only manner possible for this electric aspect to be introduced is for the transverse magnetic mass increment component of the carrying energy to be involved in a reciprocating swing, so to speak, between this magnetic state and a corresponding electric state:

$$
\mathrm{E}_{\text {total }}=\mathrm{E}_{\text {trans. }}+\left[\mathrm{E}_{\text {elec. }} \cos ^{2}(\omega \mathrm{t})+\mathrm{E}_{\text {mag. }} \sin ^{2}(\omega \mathrm{t})\right]
$$

This form of the relation then obviously leads to the following LC representation:

$$
\mathrm{E}=\frac{\mathrm{hc}}{2 \lambda}+\left[\frac{\mathrm{e}^{2}}{2 \mathrm{C}_{\lambda}} \cos ^{2}(\omega \mathrm{t})+\frac{\mathrm{L}_{\lambda} \mathrm{i}_{\lambda}^{2}}{2} \sin ^{2}(\omega \mathrm{t})\right]
$$

where

$$
\mathrm{E}_{\mathrm{B}(\max )}=\frac{\mathrm{Li}^{2}}{2} \text { and } \mathrm{E}_{\mathrm{B}(\max )}=\frac{\mathrm{Li}^{2}}{2}
$$

There was then no failing to notice the similarity between Equation (10) stemming from the Biot-Savart Equation and Equation (6) corresponding to the representation of a localized electromagnetic photon in the trispatial geometry, which led to the conclusion that the carrying energy of a moving electron can only have the same electromagnetic inner structure as that of a free moving localized electromagnetic photon; which allowed restructuring Equation (10) to incorporate the local electric and magnetic fields of the electron carrying energy as Equation (14) further on, which can indifferently be applied to the carrying energy of massive elementary particles and to free moving electromagnetic photons in replacement of Equation (6).

Further analysis then allowed mathematically demonstrating that the reason why the velocity of the electron "carrier-photon" was limited to velocities below the speed of light was uniquely due to the fact that the carrier-photon's momentum related unidirectionally oriented energy half is forced to propel the translationally inert electromagnetic invariant rest mass of the electron in addition to simultaneously propelling its own translationally inert electromagnetic other half, which can only slow it down accordingly, since the velocity of light of an electromagnetic photon is maintained in vacuum in this space geometry only due to the fact that it can only be made by structure of two equal halves, one of which remaining unidirectional while propelling the other half, which is translationally inert while electromagnetically oscillating transversally to the direction of motion, as analyzed [14].

Finally, the fact that the relativistic mass increment of an electron in motion corresponds exactly to the transversally oscillating electromagnetic half of the electron carrying energy as represented by Equation (10), and that this relativistic mass increment possesses omnidirectional inertia just like the invariant rest mass of the electron, then allowed associating the same omnidirectional inertia properties to the transversally oscillating electromagnetic half of any free moving electromagnetic photon as represented by Equations (6) and (14), which provides a direct explanation to the observed deflection angle of light grazing stellar masses, without the need to resort to the curved spacetime solution of general relativity $[12,33]$.

\section{Establishing the internal electromagnetic structure of the rest mass of localized elementary particles}

From the method used by Marmet to derive his conclusion from the Biot-Savart Equation, a new alternate general Equation for calculating the energy of electromagnetic quanta equivalent to $\mathrm{E}=\mathrm{h} v$ was then derived that does not involve Planck's constant, and is obtained by spherically integrating their energy from infinity to a lower limit that relates their longitudinal wavelength to the fine structure constant; a lower limit corresponding to the transverse amplitude $(\lambda \alpha / 2 \pi)$ of the electromagnetic oscillation of a localized photon's energy quantum in the trispatial geometry ([32], Equation (11)):

$$
\mathrm{E}=\mathrm{h}=\frac{\mathrm{e}^{2}}{2 \varepsilon_{0} \alpha \lambda}
$$

This definition of energy incidentally allows observing that Planck's quantum of action belongs to a set of electromagnetic constants that inextricably define each other: $h=\left(e^{2} / 2 \varepsilon_{0} \alpha c\right)=\left(e^{2} \mu_{o} c / 2 \alpha\right)$. Just as in the cases of Euler's identity $\left(\mathrm{e}^{\mathrm{i} \pi}+1=0\right)$ and the derivation revealing the speed of light in vacuum from Maxwell's Equations $\left(\varepsilon_{0} \mu_{0} c^{2}=1\right)$, it can be observed that the same logical rule to the effect that any value which is uniquely defined by a set of constants can only be itself a constant, now guarantees the invariance of Planck's constant.

In the present case, Planck's quantum of action can be confirmed being an electromagnetic constant by first confirming the invariance of the fine structure constant ([31], Equation (1)) with respect to three other previously established electromagnetic constants (e, $\varepsilon_{\mathrm{o}}$, and also $\mathrm{H}$, which is a newly defined electromagnetic intensity constant ([34], Equation (17))), and then confirming the invariance of Planck's quantum of action $\mathrm{h}$ ([31], Equation (4)) with respect to three previously established electromagnetic constants (e, $\varepsilon_{0}$, and a now confirmed to be invariant).

This new definition of energy in turn allowed defining the electric and magnetic fields of any localized photon, or massive elementary particle's carrying energy, from their wavelength and a specific set of known electromagnetic constants [32]:

$$
\mathrm{B}=\frac{\mu_{0} \pi \mathrm{ec}}{\alpha^{3} \lambda^{2}} \text { and } \mathrm{E}=\frac{\pi \mathrm{e}}{\varepsilon_{0} \alpha^{3} \lambda^{2}}
$$

Equations (13) applying equally to the energy quantum of free moving photons and to that of massive elementary particles' carrying energy, then allowed adapting Equation (6) to use these fields definitions instead of the less familiar and less handy capacitance and inductance definitions of Equations (7) to represent the internal electromagnetic structure of localized photons and also that of massive elementary particles' carrying energy:

$$
E \vec{I} \vec{i}=\left(\frac{h c}{2 \lambda}\right)_{X} \vec{I} \vec{i}+\left[\begin{array}{l}
2\left(\frac{\varepsilon_{0} \mathbf{E}^{2}}{4}\right)_{Y}(\vec{J} \vec{j}, \vec{J} \overleftarrow{j}) \cos ^{2}(\omega t) \\
+\left(\frac{B^{2}}{2 \mu_{0}}\right)_{Z} \stackrel{\leftrightarrow}{\mathrm{K}} \sin ^{2}(\omega t)
\end{array}\right] V
$$

where

$$
\mathrm{V}=\alpha^{5} \frac{\lambda^{3}}{2 \pi^{2}}
$$

Equation (15) determining the volume that must be associated with fields Equations (13) to implement Equation (14) is drawn from a deep analysis carried out [32] of the Equation giving the energy density associated with electric field Equation (13) when the density of both $\mathbf{E}$ and $\mathbf{B}$ fields are equal in the context of straight line motion of charged electromagnetic elementary particles:

$$
\begin{aligned}
& \mathrm{U}=\varepsilon_{0} \mathrm{E}^{2}=\varepsilon_{0}\left(\frac{\pi \mathrm{e}}{\varepsilon_{0} \alpha^{3} \lambda^{2}}\right)^{2}=\frac{\pi^{2} \mathrm{e}^{2}}{\varepsilon_{0} \alpha^{6} \lambda^{4}} \\
& =\frac{\mathrm{e}^{2}}{2 \varepsilon_{0} \alpha \lambda} \times \frac{2 \pi^{2}}{\alpha^{5} \lambda^{3}}=\mathrm{E} \times \frac{1}{\mathrm{~V}}=\frac{\mathrm{e}^{2}}{2 \varepsilon_{0} \alpha \lambda} \times \frac{1}{\left(\frac{\mathrm{a}^{5} \ddot{\mathrm{e}}^{3}}{2 \pi^{2}}\right)}
\end{aligned}
$$


It is important to note here that this volume in no way represents an actual volume of the related particle. It is by structure the theoretical stationary isotropic volume that the incompressible oscillating kinetic energy quantum would occupy if it was immobilized as a sphere of isotropic density. Metaphorically speaking, it amounts to bundling up all of the leaves in a tree into the smallest possible uniformly isotropic sphere to more easily calculate the limit volume and density of the material of which the leaves are made, which allows, in context, determining an electromagnetic particle's absolute limit density parameters, beyond which they cannot possibly be increased.

In turn, the definitions of Equations (13) allowed defining [32] the electric and magnetic fields corresponding to the invariant rest mass of the electron, separately from those of its carrying energy, by applying the electron Compton wavelength to the fields definitions of Equations (13):

$$
\mathrm{B}=\frac{\mu_{0} \pi \mathrm{ec}}{\alpha^{3} \lambda_{\mathrm{X}}^{2}} \text { and } \mathrm{E}=\frac{\pi \mathrm{e}}{\varepsilon_{0} \alpha^{3} \lambda_{\mathrm{x}}^{2}}
$$

LC Equation (6) then allowed upgrading Newton's non-relativistic kinetic energy Equation $\mathrm{K}=\mathrm{mv}^{2} / 2$ to full relativistic status by first converting it to its equivalent electromagnetic form [14]. This then allowed correcting it according to the electromagnetic structure of Equation (6) to obtain two new relativistic Equations fully derived from electromagnetism, provided as Equations (18) below; the first of which allows calculating any possible velocity state of any localized electromagnetic elementary particle from velocity zero for an electron at complete rest to the complete range of relativistic velocities of a massive elementary particle, to velocity c for free moving electromagnetic photons ([14], Equation (33a)), and the second Equation allowing calculation of the velocity of any localized massive elementary particle from the separate wavelengths of the energy of its invariant rest mass and that of its carrying energy ([14], Equation (49)); this latter more restrictive Equation being identical to Equation (55) derived from Special Relativity Equation $E=\gamma \mathrm{m}_{\mathrm{o}} \mathrm{c}^{2}$ [32]:

$$
\mathrm{v}=\mathrm{c} \frac{\sqrt{4 \mathrm{EK}+\mathrm{K}^{2}}}{2 \mathrm{E}+\mathrm{K}} \text { and } \mathrm{v}=\mathrm{c} \frac{\sqrt{4 \lambda \lambda_{\mathrm{C}}+\lambda_{\mathrm{C}}{ }^{2}}}{2 \lambda+\lambda_{\mathrm{C}}}
$$

From the product of magnetic fields Equations (13) and (17) for both the rest mass of the electron and its carrying energy, the following Equation was then established as Equation (49) [32] to obtain the magnetic field Equation for the electron in motion:

$$
\mathrm{B}=\frac{\pi \mu_{0} \operatorname{ec}\left(\lambda^{2}+\lambda_{\mathrm{C}}{ }^{2}\right)}{\alpha^{3} \lambda^{2} \lambda^{2}{ }^{\mathrm{C}}}
$$

which incidentally exactly corresponds to the magnetic field related to Marmet's Equation ([15], Equation (23)) derived from the BiotSavart Equation.

From the product of Equation (19) for the electron in motion and by Equation (18) for calculating relativistic velocities from wavelengths, electric field Equation (20) was then established for the electron in motion in straight line at any velocity. The known relation $\mu_{0} c^{2}=1 / \varepsilon_{0}$ allowed establishing this Equation in a simple manner by substitution, since the product of Equations (18) and (19) exactly corresponds to the right side of the standard Equation for calculating motion in straight line of a charged particle $\mathbf{E}=\mathrm{vB}$ stemming from the Lorent $z$ Equation:

$$
\mathrm{E}=\frac{\pi \mathrm{e}}{\varepsilon_{0} \alpha^{3}} \frac{\left(\lambda^{2}+\lambda_{\mathrm{C}}{ }^{2}\right) \sqrt{\lambda_{\mathrm{C}}\left(4 \lambda+\lambda_{\mathrm{C}}\right)}}{\lambda^{2} \lambda_{\mathrm{C}}{ }^{2}\left(2 \lambda+\lambda_{\mathrm{C}}\right)}
$$

Formal establishment of Equation (20) actually requires a complex vectorial product in the trispatial geometry, which is described [31] and that remains to be established.

\section{Mechanical explanation to $\mathrm{e}^{+} \mathrm{e}^{-}$pair production from the decoupling of $1.022 \mathrm{MeV}$ electromagnetic photons in the trispatial geometry}

Analyzing Equation (6) with respect to the greatly increased set of orthogonal geometric possibilities allowed by the expanded trispatial geometry also allows establishing a mechanical explanation to the conversion of massless electromagnetic photons to massive electron-positron pairs while preserving the cyclic oscillation of the magnetic aspect of their rest mass energy between increasing spherical presence from zero presence to maximum spherical presence, followed by decreasing spherical presence to zero presence at the electron invariant rest mass energy frequency, which is a critically important feature of the cyclic spin orientation reversal of self-sustaining localized electromagnetic elementary particles' magnetic fields brought to light in the trispatial geometry [35], which will be put in perspective further on.

Given that the total complement of energy making up the invariant rest masses of both $0.511 \mathrm{MeV} / \mathrm{c}^{2}$ particles generated possess omnidirectional inertia (electromagnetic mass) after conversion of a $1.022 \mathrm{MeV}$ electromagnetic photon, this also means that the natural conversion process allows for the unidirectional half of the photon's energy to mechanically acquire this property of omnidirectional inertia. The manner in which this is accomplished during the conversion process in the trispatial geometry, as well as how the opposite signs of the charges of both particles are acquired, is analyzed [35].

The trispatial LC Equation for the electron at rest can be formulated as follows:

$$
\mathrm{E} \overrightarrow{0}=m_{\mathrm{e}} \mathrm{c}^{2} \overrightarrow{0}=\left[\frac{\mathrm{hc}}{2 \lambda_{\mathrm{C}}}\right]_{\mathrm{Y}} \overrightarrow{\mathrm{J}} \overleftarrow{\mathrm{i}}+\left(\begin{array}{l}
2\left[\frac{\left(\mathrm{e}^{\prime}\right)^{2}}{4 \mathrm{C}_{\mathrm{C}}}\right]_{\mathrm{X}}(\overrightarrow{\mathrm{I}} \overrightarrow{\mathrm{j}}, \overrightarrow{\mathrm{I}} \overleftrightarrow{\mathrm{j}}) \cos ^{2}(\omega \mathrm{t}) \\
+\left[\frac{\mathrm{L}_{\mathrm{C}} \mathrm{i}_{\mathrm{C}}{ }^{2}}{2}\right]_{\mathrm{Z}} \stackrel{\leftrightarrow}{\mathrm{K}} \sin ^{2}(\omega \mathrm{t})
\end{array}\right)
$$

where $\lambda_{c}$ is the electron Compton wavelength. In the trispatial space geometry, the rest mass Equation for the positron is identical to Equation (21) for the electron, except for a $180^{\circ}$ orientation reversal of sub-unit-vector (i) in expression (J-i) within electrostatic Y-space, which refers to the reversal of the sign of its unit charge with respect to that of the electron [35].

\section{The Coulomb force}

Considerations on the possible origin of the momentum related translational kinetic energy that propels elementary charged particles such as electrons lead to observe that at the submicroscopic level, kinetic energy is induced in these particles exclusively as a function of the distance separating them. It is also well verified that the only known force able to induce kinetic energy in free moving charged particles is the well known Coulomb force.

Although established more than 200 years ago by C.A. Coulomb, the exhaustively confirmed Coulomb law which is in action between charged particles as a function of the inverse square of the distance separating them seems to have progressively become invisible in the background of the quantum electrodynamics method (QED), even if the Coulomb Equation is an integral part of Maxwell's first Equation, that is, Gauss' Equation for the electric field, from which it can easily be derived [13].

The Coulomb force is indeed a critically important component 
of every "virtual photon" in QED, but metaphorically cut into so many little pieces that it now attracts little attention. Metaphorically speaking, QED causes us to pay attention to every individual pixel in a metaphorical $4 \mathrm{~K}$ screen that would represent the submicroscopic level, but if we mentally pull back sufficiently, its infinitesimally progressive action can be observed again.

From observations made at our macroscopic level, the traditional concept of "force" was historically established by Newton as a mutual action between two massive bodies, in the sense that "when a body exerts a force on a second body, the second body always exerts a force on the first" ([2], p. 87). Newton established this conclusion as his third law of motion, stating that the mutual actions of two massive bodies on each other are always equal.

Considering each of these bodies separately, the force is then defined as being the interaction that changes the momentum of a body as a function of the time that this interaction is applied to it. This led to defining force as the product of the mass of a body by its acceleration, that is, its changing velocity $(\mathrm{F}=\mathrm{ma})$; and to define its momentum at any given instant as the product of its mass by its instantaneous velocity $(\mathrm{p}=\mathrm{mv})$.

This observed "apparent attraction" as a function of the inverse square of the distance between massive bodies that are not in contact with each other, then resulted in force being directly related to a natural increase in translational momentum of the body, without any immediate need to refer to the simultaneousness of the increase of its translational kinetic energy as a function of the diminishing distance between the bodies involved, which is obtained by multiplying the force by the distance between the bodies at any given moment, since acceleration is represented by the squared momentary velocity divided by the corresponding instantaneous distance $\left(a=\mathrm{v}^{2} / \mathrm{r}\right)$, which results in the total amount of energy momentarily induced in the body at this specific distance to be $\left(\mathrm{E}=\mathrm{mv}^{2}\right)$, a total amount of induced kinetic energy that Leibnitz considered the real effect of application of a force, as mentioned previously ([2], p. 222), quantity which incidentally is twice the amount associated with the translational momentum (p), which on its part is traditionally calculated by replacing $(\mathrm{v})$ by $(\mathrm{p} / \mathrm{m})$ in the classical kinetic energy Equation $\left(\mathrm{K}=\mathrm{mv}^{2} / 2\right)$, giving $\left(\mathrm{K}=\mathrm{p}^{2} / 2 \mathrm{~m}\right)$ ([1], p. 134).

From the relativistic perspective, the reason for the difference between these two energy measuring methods is that $\left(E=\gamma m_{o} v^{2}\right)$ also includes the induced energy that converts to the velocity related momentary relativistic mass increment that was transversally measured by Walter Kauffman when he deflected relativistically moving electrons in a bubble chamber at the turn of the $20^{\text {th }}$ century [6], and that was established by Paul Marmet as corresponding to the relativistic magnetic mass increment as represented in Equation (8), while $\left(\mathrm{K}=\gamma_{\mathrm{m}} \mathrm{v}^{2} / 2\right)$ provides only the correct amount of momentum related translational kinetic energy that sustains the velocity of the total relativistic mass, that is, an amount of unidirectional kinetic energy that turns out by structure to correspond to exactly half of the total amount of kinetic energy that must be induced in the electron in excess of its invariant rest mass energy for it to move at the corresponding relativistic velocity, as analyzed [14], and represented in Equation (8).

It must be put in perspective that these definitions, quite useful at our macroscopic level when applied to massive macroscopic bodies, were established before it was discovered that the force in action between charged elementary particles actually induces kinetic energy in these particles due to the fact that they are electrically charged, so in the absence of this information discovered later, the same definitions of force and momentum were applied by default to the Coulomb force as applicable to these elementary massive subcomponents of atoms, without taking into account that besides their mass, they also possess an electrical charge, which is precisely the characteristic related to energy induction in electromagnetism.

The Coulomb force was thus defined in the following manner:

"The force of attraction or repulsion between two point charges is directly proportional to the product of the charges and inversely proportional to the square of the distance between them." ([3], p.462).

But deep analysis of the Coulomb force in light of the internal electromagnetic energy structure of the carrying energy amounts induced in charged particles such as electrons and positrons revealed in the trispatial geometry, and of the variation of these amounts as distances vary between charged particles, reveals that the force itself does not directly attract nor repel in the manner that it is currently defined to operate, but that it only adiabatically induces kinetic energy in electrically charged elementary particles, and that it is the unidirectional momentum related component of this adiabatic kinetic energy that vectorially orients itself to cause charged particles to translationally tend to move toward each other in case of opposite signs charges, or away from each other in case of same sign charges, when the particles are not captive in the various stable electromagnetic resonance equilibrium states allowed in atomic structures, states into which this translational motion is hindered even if the momentum related kinetic energy still remains adiabatically induced, as analyzed [33]. This adiabatically maintained presence of kinetic energy will be analyzed further on.

This brings to light that the Coulomb force would not really be a "force of attraction or repulsion" as traditionally defined, but would rather be a "force of adiabatic kinetic energy induction" that would adiabatically and continuously induce kinetic energy in elementary charged particles, whether they are moving or not, which would make this force a "yet-to-be-correctly-understood-active-agent" that would be universally ambient in the background, so to speak, and consequently that it would not need to travel at any velocity to simultaneously act on all existing charged particles in the universe, but would only increase or decrease the amounts of this adiabatically induced kinetic energy in an infinitesimally progressive manner whenever charged particles happen to be in distance varying motion with respect to each other.

Moreover, Marmet's discovery and the observation confirmed by the Kaufmann experiment that half of any carrying energy quantum induced in electrons converts to mass, reveal that not only does the Coulomb force induce the momentum related translational energy of elementary charged particles, it also induces actual mass, made up of the electromagnetically oscillating other half of the induced carrying energy, as represented with Equations (8) to (10) and as established in references $[5,14,32]$.

From this perspective, and given that this carrying kinetic energy needs to be induced in charged particles "before" any related motion can become possible, this means that no motion of the charged particles is required for the Coulomb force to adiabatically induce kinetic energy in them as a function of the distance, and that this energy remains induced even if the related velocity is prevented from being expressed when the particles are captive in stationary orbital resonance states, which are states of induced momentum kinetic energy that the classical concept of momentum, thus also of the Lagrangian and the Hamiltonian, clearly do not account for since its related translational 
velocity is then forcibly reduced to zero, or averages out to zero for electrons captive in such axial resonance states.

Also, the currently accepted conception is that the Coulomb force would be in action in the hydrogen atom between the electron and the "proton". This conclusion disregards the fact that the proton is not an elementary charged particle, but a system of elementary charged particles, just like the solar system not a single body, but a system of smaller massive astronomical bodies.

Regrettably, 50 years after that this major discovery was experimentally confirmed at the Stanford linear accelerator in 1968 [18], it seems that few introductory textbooks to particle physics clearly mention this discovery with proper reference, but instead continue referring to protons and neutrons as being elementary particles, which induces a high level of confusion in the community in this regard.

Obviously, the solar system is a system whose internal structure is defined by planets stabilized on orbits about a central star, and just as obviously since the 1960's, the proton is known to be a system whose internal structure is defined by interacting elementary particles that are charged, massive, scatterable and point-like behaving just like the electron, that were named up quark and down quark, that are electromagnetically stabilized into least action equilibrium resonance states.

So since the Coulomb force can act only between electrically charged particles, it obviously can be interacting only between the charged electron and the charged up and down quarks that are captive inside the proton structure. So these 3 particles are the only stable interacting charged and massive elementary particles that can be identified as the physical building blocks of all atoms in the universe, instead of the three that are still often erroneously referred to as being the three fundamental elementary particles set defining the inner structure of atoms: electron, proton and neutron.

Consequently, from the electromagnetic perspective, the hydrogen atom is not an interacting two-massive-body system as it still is currently considered, but rather a four-charged-electromagnetic-particle system stabilized in least action electromagnetic resonance states.

In light of these considerations, a tentatively more precise definition of the Coulomb force could be formulated in the following manner, for example:

"The Coulomb force adiabatically and continuously induces kinetic energy in elementary charged particles as a function of the inverse square of the distance separating them, thus inducing in each charged particle an accompanying energy quantum whose unidirectional half is vectorially oriented so that charged particles tend to close in on each other if they have opposite signs charges, and move away from each other if they have identical sign charges, when not captive in the various resonance states allowed in atoms, and to apply pressure in these vectorial directions when their motion is inhibited by local electromagnetic equilibrium states." [33].

So from the submicroscopic perspective, it would then appear that it is not the macroscopic bodies themselves that are subject to a force, but the individual charged and massive point-like behaving electromagnetic elementary particles whose sum of masses makes up the total masses of macroscopic bodies, and that the only force that can act on them would be by structure the so-called "Coulomb force", which would then not be an attractive and repulsive force as initially defined by similarity with the apparent inverse square attraction force between macroscopic masses that was the only possible interpretation in Newton's time, but would rather be an underlying "adiabatic-kinetic- energy-inducing-yet-to-be-correctly-understood-active-agent", that we name the "Coulomb force", which could be by very nature permanently and statically present in the universe and in permanent action between all charged elementary particles in existence.

This means that the kinetic energy induced in any pair of charged particles is inversely proportional to the distance separating them irrespective of the time elapsed, if they are maintained at a fixed distance from each other, and that it adiabatically varies in both particles if they are in motion relative to each other, irrespective of their relative velocity and irrespective of the time elapsed during the corresponding motion sequence.

In the trispatial geometry, both neutral internal charges of electromagnetic photons would logically acquire opposite vectorial signs on the $\mathrm{Y}-\mathrm{y} / \mathrm{Y}-\mathrm{z}$ plane, but would both appear neutral along the perpendicularly oriented $\mathrm{Y}-\mathrm{x}$ axis along which they do not travel, this latter apparently neutral state being the state observable from the perspective given us from within normal X-space in the case of electromagnetic photons.

This tentatively reformulated definition will now allow describing the Coulomb force adiabatic kinetic energy induction process at play within atoms between the charged and massive elementary particles that they are made of.

However, to simplify the description, the traditional terms of "attraction" and "repulsion" will continue to be used often in this text, but always keeping in mind that "attraction" refers to unidirectional carrying energy being vectorially oriented toward an opposite sign particle, and that "repulsion" refers to unidirectional carrying energy being hindered in its translational motion.

\section{Adiabatic kinetic energy induction in atomic and nuclear structures}

Analysis of the manner in which temperature adiabatically increases with depth inside the Earth mass [36] leads to conclude that this increase can only be related to a progressive compression increase with depth of the electronic orbitals about the nuclei of the atoms making up the mass of the Earth, that would shorten the mean distances between the electrons and the up and down quarks that are the only elementary charged sub-components of the nucleons making up these nuclei, which can only increase the amounts of kinetic energy induced in them by the Coulomb force as a function of the inverse square of these shortened distances.

In turn, this leads to observe that for electrons stabilized into such natural least action states, the kinetic energy can only be induced in an adiabatic manner, since this energy varies progressively as distances vary between these charged particles without any of it being emitted to the environment or being contributed by the environment during this natural compression driven distance variation process [5].

Since all three elementary massive particles that can be detected via non-destructive scattering [31] within all atoms in existence (electrons, up quarks and down quarks) are charged, this of course means that adiabatic kinetic energy is permanently induced in all of them, whose quantities are clearly measurable for the stable average axial resonance distances that separates them, and that necessarily correspond to the electronic orbitals for electrons, and to nucleonic orbitals for up and down quarks inside nucleons.

An extensively documented case of such a level of adiabatic carrying energy induction by the Coulomb force is that of the ground state orbital of the hydrogen atom: 


$$
\begin{aligned}
& \mathrm{E}=\int_{\mathrm{a}_{0}}^{\infty} \frac{1}{4 \pi \varepsilon_{\mathrm{o}}} \frac{\mathrm{e}^{2}}{\mathrm{r}^{2}} \cdot \mathrm{dr}=0-\frac{1}{4 \pi \varepsilon_{\mathrm{o}}} \frac{\mathrm{e}^{2}}{\mathrm{r}_{\mathrm{o}}} \\
& =-4.359743805 \mathrm{E}-18 \mathrm{~J}(27.2 \mathrm{eV})
\end{aligned}
$$

where $\left(r_{0}\right)$ is the Bohr radius, which exactly corresponds to the mean distance separating the electron, stabilized in axial resonance state in the ground state orbital, from the charged up and down quarks captive in the central proton of the hydrogen atom.

The previously established internal electromagnetic structure of the carrying energy of the electron described by Equation (14) now reveals that half of this adiabatic energy systematically converts to a mass increment, possessing omnidirectional inertia just like the invariant rest mass of the electron, that increases the momentary electron mass, whether the electron is translationally immobilized in this manner, or freely moving at the velocity corresponding to this amount of carrying energy, as confirmed by Kaufmann's transverse mass measurements of electrons moving at relativistic velocities [6].

This measurable state can now be directly related to the difference between the total amount of carrying energy provided by Equation $\left(E=\gamma m_{0} v^{2}\right)$ stemming from acceleration Equation $\left(F=\gamma m_{o} a\right)$, and half this amount calculated with Equation $\left(\mathrm{K}=\gamma \mathrm{m}_{\mathrm{o}} \mathrm{v}^{2} / 2\right)$, that provides only the translational momentum related kinetic energy that propels the total relativistic mass of the particle.

Since the same adiabatic kinetic energy inducing Coulomb force is structurally at play between the charged up and down quarks inside the proton, the adiabatic mass increments due to their immensely higher levels of carrying energy can only be much more important than in even the most energetic electronic orbitals, given the extremely short distances separating them within nucleons' structures.

Close study of nucleons' structures in the frame of the trispatial geometry in light of the unavoidable presence of this permanently induced adiabatic energy, that contributes to increase the mass of elementary particles as a function of these very short axial distances between the up and down quarks, then led to the establishment of trispatial LC Equations for the rest mass energy and for the carrying energy levels of these elementary charged and massive particles making up the internal structure of nucleons that are consistent with observation $[5,13,37]$

These Equations reveal that the carrying energy level reached for each up and down quark within the proton structure is about 600 times higher than the energy contained in the rest mass of the electron stabilized in the ground state orbital of the hydrogen atom [13].

\section{The cyclic polarity reversal of elementary particles magnetic fields}

The oscillating nature of the magnetic component of elementary particles' invariant rest mass energy and also that of their carrying energy as revealed by LC Equations (14) and (21), makes obvious that, in the trispatial geometry, the physical presence of this magnetic component can only oscillate between zero presence and maximum spherical presence in space and then back to zero presence at the frequency and to a physical spherical extent related to the amount of energy contained in their quanta.

In the trispatial geometry, a "point-like junction area" or "point-like passage area" is located at the center of each localized electromagnetic quantum, which allows its energy to freely circulate between the three thus interconnected spaces as if between communicating vessels, and
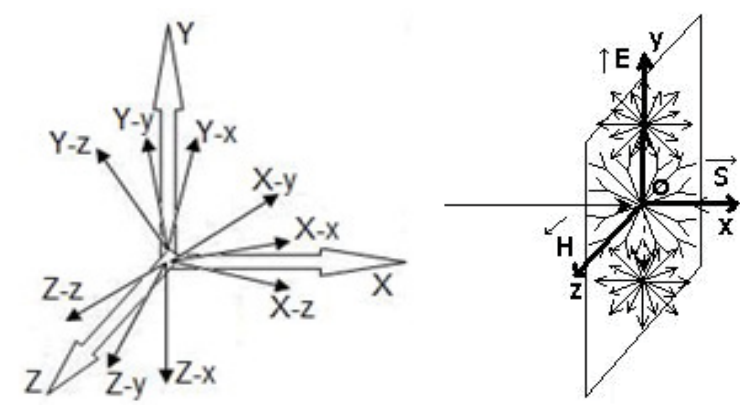

Figure 1: The orthogonal structure of the 3-spaces geometry complex, and plane wave reference frame applied to a permanently localized photon.

to locally stabilize in a state of self-sustaining dynamic equilibrium between the three 3-dimensional orthogonal spaces constituting the trispatial geometric complex within which each electromagnetic energy quantum exists (Figure 1), which is completely described in references $[12,31]$, and that allows the energy of the quantum to be described as one unidirectional half remaining in translational momentum orientation within normal X-space for the photon, while the other half electromagnetically oscillates transversally between two separate orthogonal 3-dimensional spaces that are perpendicular with respect to each other and with respect to normal X-space, one of which is identified as $\mathrm{Y}$-space, allowing manifestation of the properties represented by the electric $\mathbf{E}$ field, while the other is identified as Z-space, allowing manifestation of the properties represented by the magnetic $\mathbf{B}$ field. This latter half of the particle's energy, being longitudinally inert by structure, consequently displays omnidirectional inertia by definition in normal X-space, that is, "electromagnetic mass".

In the trispatial geometry, this point-like junction is meant to represent the observable and measurable "point-like behavior" of charged elementary particles such as photons or electrons during scattering encounters between these particles in normal space.

In this space geometry, the energy making up the magnetic component of the rest mass of the electron is by structure in constant internal motion, successively in two opposite spherical orientations, from an initial state of zero presence within magnetostatic Z-space at the beginning of each cycle, after having completely transferred into another space of the complex, this motion of the energy will then consist in two distinct phases, the first being a spherical expansion phase as it omnidirectionally enters Z-space through the point-like junction, until maximum radial expansion has been reached. The second phase will consist in a reverse motion inwards through the trispatial junction as an omnidirectional spherical regression of this energy until it has completely evacuated Z-space. This oscillation process redefines the spin of an elementary electromagnetic particle as becoming a property relative to the state of the expansion and regression cycles of the presence of the magnetic energy of all other electromagnetic elementary particles.

This behavior also implies that both poles of the magnetic field of an electromagnetic elementary particle have to geometrically coincide by structure with the location of the trispatial point-like junction located at their center. This means that a relative parallel spin alignment between two electrons will occur, for example, when the magnetic presence of the energy of both particles is synchronously in spherical expansion and regression at the same time, which amounts to a spherical inverse cube magnetic repulsion with distance between both magnetic spheres, since the magnetic energies of both particles remain vectorially opposing 
each other during the whole sequence; while relative antiparallel spin alignment will occur when the magnetic energy presence of one electron is synchronously in its expanding spherical presence phase while that of the other electron is in its regressing spherical presence phase, which amounts to a spherical inverse cube magnetic attraction with distance between both particles, since the energies of both spherical magnetic spheres remain vectorially moving in converging directions during the complete sequence.

Interestingly, the resulting magnetic inverse cube interaction between two electrons forced to interact in parallel repulsive spin orientation was recently experimentally measured by Kotler et al. in 2014 [38].

Moreover, the force that can be calculated between both electrons from the data collected, whose analysis results in the establishment of Equation (23), amounts to exactly half the force that can be calculated from the magnetic interaction between two bar magnets within each of which both north and south poles are by structure separated by a measurable distance, and whose force between their simultaneously interacting 2 pairs of poles is calculated with Equation (24), which is the standard Equation established for dealing with bar magnets ([2],p 93).

$$
\begin{aligned}
& F=\frac{3 \mu_{0} \mu^{2}}{4 \pi d^{4}} \\
& F=\frac{3 \mu_{0} \mu^{2}}{2 \pi d^{4}}
\end{aligned}
$$

This difference in intensity of the force calculated with these two Equations appears to directly relate to the fact that within a pointlike behaving electromagnetic particle, in which the distance between both poles reduces to zero by structure, both poles can only exist in alternance one at a time in succession, which directly correlates both poles, as well as the relative spin of the particle, to both magnetic energy presence increasing and decreasing phases of the oscillating electromagnetic cycle of the quantum's energy as described by the trispatial LC Equations.

Interestingly, the alternating presence of both magnetic poles for magnetic fields for which both poles geometrically coincide like those seemingly observed for the point-like behaving electrons in the Kotler et al. experiment can be quite easily confirmed at our macroscopic level with circular magnets magnetized parallel to thickness, such as loudspeaker magnets [37].

Due to the need for the loudspeaker coil to constantly tend to keep perfect perpendicular axial alignment in the central hole of these magnets, this orientation of the magnetic field during the magnetization process forces both poles of the macroscopic magnetic fields that develop about them to geometrically coincide by structure at their geometric center, the proof being that from the data collected from the interaction of such mutually interacting magnets, the force that can be calculated systematically obeys Equation (23) just as for the electrons of the Kotler et al. experiment, and cannot be made to obey Equation (24) under any circumstance, as analyzed [37], thus demonstrating that during interaction between two such magnets for which both magnetic poles coincide by structure within the magnetic field of each magnet, or in context, between the two point-like behaving electrons of the Kotler et al. experiment, only two poles at a time are simultaneously interacting, and never 4 poles as with bar magnets.

A surprising conclusion of this observed and measured behavior is that magnetic fields inside which both poles geometrically coincide can only be monopolar at any given instant, which means that the magnetic field of the invariant rest mass of electrons as described in the trispatial geometry, and as measured during the Kotler et al. experiment, is a magnetic monopole by structure at any given instant.

Indeed, the Kotler et al. experiment and the circular magnets experiment demonstrate out of any possible doubt that only 2 magnetic poles at a time can simultaneously be interacting during such magnetic encounters between magnetic fields such as those of electrons, that is, only one pole at a time belonging to each particle, which appears to completely validate the cyclic magnetic spin reversal process mandated by the inner structure of electromagnetic particles in the trispatial geometry.

\section{Magnetic fields interaction as a function of identical oscillating frequencies}

This state of cyclic magnetic polarity reversal of the magnetic component of the electron brings an entirely new light to the reason why two electrons can associate in antiparallel spin alignment to fill electronic orbitals or to associate in covalent bounding between atoms, despite their electric repulsion as a function of the inverse square of the distance separating them, on account of their identical electrical sign, which at first glance should logically prevent such close association of two electrons.

The answer obviously lies in the fact that their magnetic fields interact as a function of a higher order interaction law than the inverse square electric interaction law (Figure 2), which means that when forced by local electromagnetic circumstances to come close enough to each other for the inverse cube magnetic interaction to start overcoming the inverse square interaction, they will easily switch to mutually attractive antiparallel spin alignment, which is a least action state with respect to parallel magnetic spin alignment. This process is analyzed [5].

The same process also explains why a pair of electron and positron that mutually capture in metastable positronium configuration always succeeds in actually spiralling inwards until they meet and convert to electromagnetic photons states as the systematic final stage of the positronium decay process, that benefits from the additional favorable circumstance that contrary to a pair of mutually interacting electrons, both particles also electrically attract as a function of the inverse square law, which easily brings them to the equilibrium point at which the inverse cube magnetic interaction will dominate [5]. Their respective amounts of carrying energy being equal by structure in the positronium

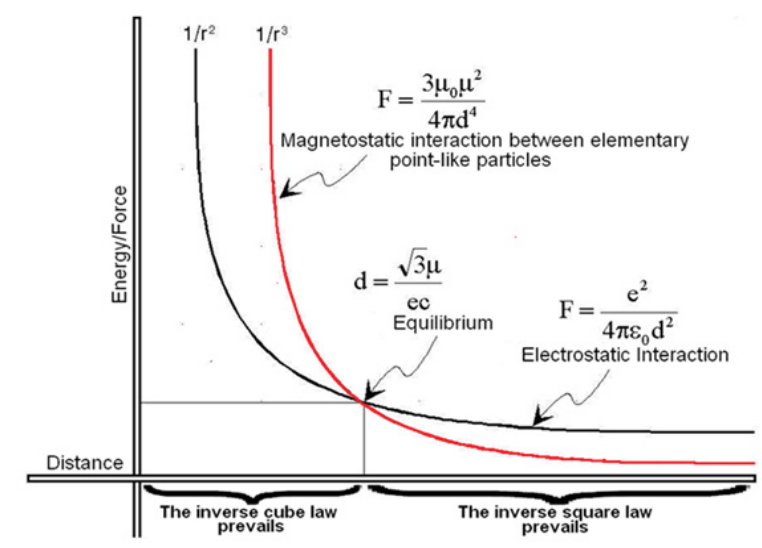

Figure 2: Intersecting inverse square and inverse cube interaction curves. 
system, their contributing magnetic fields will also oscillate at the same mutual frequency and will not hinder the process in any way.

The success of the antiparallel spin coupling of electron pairs in covalent bounding and electronic orbital pair filling, as well as the final stage of the positronium decay process resulting in the electron and the positron physically joining to convert to electromagnetic photons state is intimately linked in the trispatial geometry to the fact that the magnetic oscillating frequencies of both particles are identical, which allows them to easily fall into perfectly synchronized least action antiparallel magnetic oscillation.

\section{Magnetic fields interaction as a function of different oscillating frequencies}

The situation is quite different however when an electron and a proton are interacting to form a hydrogen atom, even though they display equal intensity opposite charges signs similar to those of an electron-positron pair meta-stabilizing into positronium configuration.

The difference lies with the apparent unit positive charge of the proton system, which, let us recall, is a system of elementary particles electrically charged, and is not itself a charged particle. The peculiarity with the proton apparent unit charge, which is often overlooked, is that its assumed unit charge is the results of the addition of the fractional charges of its three elementary components, that is, $+2 / 3+2 / 3-1 / 3$ $=+1$. So this means that the electron is not really electromagnetically interacting with the proton as such, but rather with its three electromagnetic elementary charged inner subcomponents (uud).

Contrary to the positronium case, where both particles' magnetic energy oscillates at the exact same frequency in the trispatial geometry, the hydrogen atom involves the frequencies of the two oscillating magnetic fields of the electron and of its carrying energy on one hand, which are now interacting with the much higher oscillating frequencies of the magnetic fields of the charged inner sub-components of the proton and of their immensely more energetic carrying energy on the other hand $[5,13]$.

In the best of cases, the magnetic polarity reversal of the magnetic presence of the most energetic proton inner components occurs more than 600 times during each magnetic presence cycle of the electron magnetic energy (Figure 3), which, due to the fact that the intensity of the inverse cube magnetic interaction force drops rapidly with increasing distance, results in the magnetic interaction between the electron and the proton inner components becoming predominantly repulsive each time the electron comes closer to the proton than the mean ground orbital distance, which happens to correspond to the distance at which the intensities of both the electric force and the magnetic interaction fall into equilibrium (Figure 2).

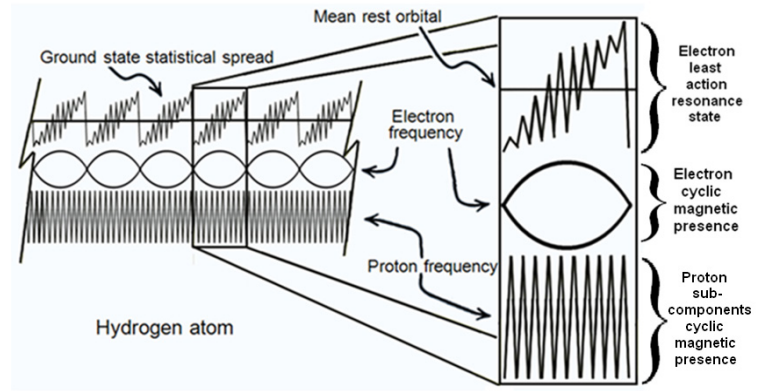

Figure 3: Establishment of the least action resonance state of the electron in the hydrogen atom.
The constant interplay due to the different frequencies of the various magnetic fields involved in inverse cube interaction that oppose the unidirectional momentum energy of the electron that constantly tends to cause the electron to move toward the proton can then only result in the establishment of a stable axial resonance state (Figure 3) that certainly can be related to Louis de Broglie's initial intuition that electronic orbitals have to be such resonance states, which in the trispatial geometry correspond to the various least action electromagnetic equilibrium states into which elementary charged particles become captive within atomic and nucleonic structures [5].

The detailed foundation of the mechanics of this electromagnetic resonance state is analyzed in references $[5,13]$, and can be summarized as follows. Considering Figure 3, the central sequence represents an arbitrary sample of 6 occurrences of the intensity variation of the spherical presence of the electron magnetic energy as a function of its frequency. In a simplified manner, each of these 6 occurrences is symbolically confronted in the lower sequence by the more than 600 occurrences of the spherical intensity variation of the presence of the magnetic energy of only one of the carrying energy quanta of the up and down quarks of the proton as a function of its own frequency.

Figure 3 represents the fact that while the electron reverses its spin polarity once, this inner component of the proton reverses its own spin polarity more than 600 times, which means that during each electron spherical magnetic energy presence cycle, the magnetic field of this proton inner component will alternate more than 600 times between being in relative parallel spin alignment with respect to the electron's magnetic field spin orientation, thus repelling it, and being in relative antiparallel spin alignment, thus attracting it.

The least action orbital equilibrium state is consequently established by the fact that the permanently induced unidirectional translational momentum component of the adiabatic carrying energy of the electron, that constantly tends to propel the electron toward the proton, is alternately hindered in its forward motion each time the magnetic interaction function of the inverse cube law becomes repulsive, causing both magnetic spheres involved to repel each other, and is then freed from this counter pressure while the magnetic interaction becomes attractive.

As represented with Figure 3, during each of the 600 magnetic cycles of the proton inner component, the electron will be axially repelled away from the proton by distance "d" during half of the proton inner component magnetic presence cycle during which the spin alignment is parallel, and since the electron will be farther away from the proton as the relation becomes antiparallel for the same duration, there will be a physical impossibility for it to be axially brought back all the way to distance "-d", given that the inverse cube force will be weaker at this farther location from the proton at the beginning of the antiparallel phase.

Therefore, by structure, due to the more weakly acting inverse cube attraction at the beginning of attractive phase, the electron can be axially brought back only to distance "-(d- $\Delta \mathrm{d})$ ", which will cause it to progressively move away from the proton at each polarity reversal sequence until its own magnetic energy presence falls to zero, moment at which only the electron adiabatic carrying energy translational momentum energy will be at play, causing the electron to move as close to the proton as the inverse square law will bring it until its next magnetic presence cycle initiates and that the whole predominantly repulsive magnetic sequence is initiated again, as represented with Figure 3. 
Of course the actual least action orbital resonance state of the electron in the hydrogen atom or in any other atom will be much more complex than hinted at with this limited example, which is only meant to describe the fundamental mechanics of the process, and will mandatorily involve all such electromagnetic interactions between the magnetic field of the electron and those of all other electromagnetic components captive into nearby atomic and nuclear structures.

Given the mean equilibrium distance that this process causes the electron to stabilize at in the hydrogen atom, it also becomes obvious that the probability distribution of all of the possible instantaneous locations that the electron will stochastically visit about this mean axial distance will be similar to Heisenberg's statistical distribution and will be restricted within axial limits consistent with the fact that the actual amplitude of the volume that the electron can thus visit is dependent on its varying relativistic mass related inertia at any given instant $[5,13]$ :

$$
\int_{-\delta}^{+\delta}|\psi|^{2} \mathrm{dxdydz}=1
$$

It seems also entirely reasonable to conclude that the elementary charged up and down quarks making up the scatterable inner structure of protons and neutrons and their carrying energy, which are the only constituting subcomponents of all atomic nuclei in the trispatial geometry, as analyzed [13], would be subject to similar resonance states within their own local least action electromagnetic equilibrium states, that could then also potentially be described by the various methods of quantum mechanics.

\section{Resonancestatesinquantum mechanics and electromagnetism}

It can be observed that quantum mechanics and electromagnetism deal with resonance states from entirely different perspectives, the first at the general level by means of the wave function, that establishes resonance volumes, as for example in a simpler manner in classical mechanics to calculate the volume of space visited by a vibrating guitar string; and the second more directly from the reciprocating mutual induction of electric and magnetic fields as embodied with LRC resonance for example. This is why it appears entirely logical that the inner LC electromagnetic structures that the trispatial geometry allows associating with electromagnetic elementary particles, that allows associating a permanent localization of the moving electron by relating the internal point-like junction of the LC behavior of their energy quantum to their point-like behavior in all scattering encounters, could thus allow the description of the actual electron axial resonance trajectory mechanics within the volumes defined at the general level by the wave function, which, when properly mathematized, could thus provide a fourth quantum mechanics representation that will reconcile permanent localization of the electron with the wave function.

Other studies can also be located that endeavor to directly correlate $\mathrm{QM}$ and electromagnetism from the resonance perspective. One example is this interesting study by Golovko [39] regarding resonance interactions between QM stationary states and electromagnetic wave emission and absorption.

90 years after the identification by Louis de Broglie that electronic orbitals have to be resonance states [24], research on resonance states seems to be resuming in new directions. Another example is this fascinating study on resonance states in the solar corona by Antony Soosaleon involving electric and magnetic fields interaction [40], which proposes a solution to the currently unexplained extreme heat in the solar corona, which is different from that which naturally stems from the trispatial geometry as proposed [41].

\section{Momentum, the Hamiltonian and the Lagrangian}

As mentioned previously, an analysis from the electromagnetic perspective reveals that the progressive adiabatic heat increase with increasing depth in the Earth mass can be related only to an adiabatic compression gradient of the electronic orbitals toward the nuclei of the atoms making up the mass of the Earth as depth increases [5]. This process mandatorily involves an increase in adiabatic kinetic energy induced by the Coulomb force in all electrons stabilized in the various orbitals, due to the related shortening of the inner atomic axial distances separating them from the nuclei of the atoms to which they belong.

Going back to the origin of the concept of momentum, it can be observed that the concept was intimately tied to motion before the existence of electrically charged and massive elementary particles was discovered and the Coulomb force identified as being the ultimate cause of kinetic energy induction in them, as previously put in perspective.

Although adiabatic processes were already being studied at the time, the idea that momentum related translational kinetic energy could remain induced in bodies stabilized into natural least action dynamic equilibrium states might be related to such adiabatic process obviously did not attract attention, such as the stabilized momentum energy of the massive elementary particles making up the mass of the Earth on its orbit about the Sun.

The initial concept of the presence of kinetic energy as being dependent on motion was then not re-visited and was integrated unchanged into the representations by means of the Lagrangian and then of the Hamiltonian to be applied at the submicroscopic level, even after incorporation of the concept of electromagnetic fields, which perpetuated the assumption that translational motion needs to occur before kinetic energy could even exist and for the related magnetic and electric fields to emerge at the submicroscopic level, instead of concluding that kinetic energy mandatorily had to initially adiabatically exist before motion and the related electric and magnetic fields could emerge from its presence.

This led to the still current perception that momentum related kinetic energy has to convert to "potential energy" so that the process can be seen as conservative, when the motion of electrons is hindered when captured in into atomic structures, which disregards the fact that in physical reality, this momentum related kinetic energy remains adiabatically induced in these electrons even when their motion is inhibited.

It seems that in reality, this adiabatically maintained but translationally hindered momentum kinetic energy continues to "fight" against this hindrance, a constant fight that manifests itself as a permanently maintained axial "pressure" in the vectorial direction of the nucleus against the counter-pressure of the predominantly repulsive magnetic interaction between the magnetic energy of the electrons and that of the inner components of the nucleons of which atomic nuclei are made.

Consequently, contrary to the current momentum conservation concept expectations, it would seem that momentum related kinetic energy would be a really physically existing "substance" and that it would behave accordingly. This means that it would not "miraculously" morph into becoming some form of inactive nondescript characteristicsless potential energy when the motion that it sustains is hindered, to just as "miraculously" morph back into becoming active unidirectional kinetic energy when its motion is unhindered as currently represented, but would rather remain constantly present and active even when 
its motion is hindered, but in a manner that the current concept of momentum/Lagrangian/Hamiltonian is unable to account for.

The consequence of this concept of conservative momentum having been carried on into the Lagrangian and the Hamiltonian without being adapted to account for this fact, is that with respect to the relations between force, motion and matter, classical and relativistic mechanics (CM and RM) keep on dealing with this "real kinetic energy" almost as an afterthought, due to the fact that in CM and RM the only parameter determining momentum besides mass is velocity. Since mass is defined as remaining constant in CM and RM, this makes kinetic energy appear as being an emergent quantity that depends on the prior presence of velocity, and not as a pre-existing primordial quantity that can cause velocity when its motion is not hindered by local electromagnetic circumstances.

In reality, the adiabatic nature of the kinetic energy induced in charged particles rather mandates that in reality, velocity, pressure, charge and mass, can only be emergent properties due to the adiabatically maintained presence of this kinetic energy. Of these four properties of kinetic energy, pressure and the sign of charges are related in the trispatial geometry to the forced inhibition of the translational velocity of the momentum related unidirectional kinetic energy half quantum of elementary electromagnetic particles and of their carrying energy, forcing this unidirectional kinetic energy into configurations that induce these properties; while mass, more precisely defined as being "omnidirectional inertia", is related to the fact that the transversally oscillating electromagnetic half quantum of any photon or elementary particle carrying energy, and the whole quantum of massive elementary particles, are translationally inert in normal space $[1,31]$.

It may well be the fact that the kinetic energy of a body is considered to fall to zero when this body is translationally immobilized, in the Hamiltonian/Lagrangian traditional conservative conception of momentum, that made it difficult up to now to clearly identify the nature of these three last properties of kinetic energy, since they seem to be linked to this adiabatically maintained presence, accompanying the invariant rest mass of all massive and charged elementary particles making up all macroscopic massive bodies, of quantities of unreleasable kinetic energy not subject to the Principle of conservation of energy [5], and of which the Hamiltonian and Lagrangian, as currently defined, are unable to account for when translational velocity falls to zero, or averages out to zero during such axial resonance states of motion.

\section{The submicroscopic momentum disconnect}

It can also be observed that there is a major difference between the definition of momentum applied to classical and relativistic mechanics on one hand, and that applied to electromagnetism, QED and QM on the other hand. This difference relates to the fact that the first two were developed to deal with physical processes at the macroscopic level without taking the electromagnetic properties of elementary particles into account, while the second group was developed to deal with the physical processes at the submicroscopic level of physical reality where there is no choice but to take these properties into account, despite some overlap of both levels by relativistic mechanics and electromagnetism.

What characterizes the first group is that it deals strictly with masses and their observed interactions, mainly at the macroscopic level, without taking into account that their measurable mass at the macroscopic level is only the result of the addition of the individual invariant masses of the charged elementary particles of which they are made and of the massive components of their carrying energy that physically exist at the submicroscopic level. The second group on its part directly deals with the electrically charged electromagnetic elementary particles and their carrying energy without taking into account that the electromagnetic energy that they are made of can exist only in the form of localized self-sustaining quanta involving mutual electric and magnetic induction, which is the fundamental requirement for electromagnetic energy to even exist in electromagnetic theory.

At this submicroscopic level, it was clearly established that the only way for an electron, charged and massive, to be translationally stopped in nature with respect to its environment is for it to be captured by an atom into one of the electromagnetic resonance states that are permitted in this atom; which involves, besides losing its accumulated translational kinetic energy half-quantum as an escaping bremmsstrahlung electromagnetic photon, which is subject to the Principle of conservation of energy, the simultaneous adiabatic induction of the exact same amount of replacement translational kinetic energy, that should also be related to the concept of momentum/ Lagrangian/Hamiltonian, mandated by the Coulomb force at this distance from the nucleus, as put in perspective [5], that immediately and synchronously replaces the emitted energy, even if its now hindered translational velocity toward the nucleus now averages out to zero, which is an amount of translationally hindered kinetic energy that will nevertheless remain induced in the particle for as long as the particle will remain in this related least action resonance state.

In all such cases, instead of converting to inactive virtual "potential energy" as currently assumed with the traditional concept of momentum/Hamiltonian/Lagrangian, when the translational velocity of elementary charged particles is hindered, the induced kinetic energy can only remain active, applying continuous "pressure" in the same vectorial direction.

The consequence of the current definition of momentum as being conservative is that in all domains of conventional physics, that is, classical and relativistic mechanics, electromagnetism, electrodynamics and quantum physics, kinetic energy is deemed to exist only if translational motion occurs for a mass at the macroscopic level and for a charged and massive elementary particle at the submicroscopic level, and is viewed by structure as non-existent when translational velocity is reduced to zero, which is where there is such an irreconcilable disconnect between the traditional concept of momentum/ Hamiltonian/Lagrangian and the real state of adiabatic kinetic energy induction in all electrically charged elementary particles captive in least action electromagnetic equilibrium states at the submicroscopic level.

\section{Diabatic and adiabatic processes}

Few studies have been carried out regarding adiabatic processes at the submicroscopic level that could be related to the Hamiltonian, and all of them involve changes of state due to changes in ambient conditions as a function of time. These time based changes are covered by the adiabatic theorem that was established by Max Born and Vladimir Fock in 1928 [42]. It is to be noted that these conclusions have not been re-visited since, and that no traceable study seems to have been carried out after the confirmed discovery that nucleons are not elementary particles, but are complex systems made of charged and massive elementary particles also stabilized into least action electromagnetic equilibrium states exactly like electrons in their orbital states.

The Born-Fock analysis concluded that rapid changes in ambient conditions (varying ambient magnetic fields, for example) prevent systems from adapting their configurations, which causes them to 
remain unchanged, processes that they termed "diabatic processes", leaving the final Hamiltonian in a state equivalent to its initial state.

Alternately, they concluded that gradual changes of ambient conditions allow systems to adapt their configurations, which results in their probability densities to be modified during these processes, termed "adiabatic processes", causing their final Hamiltonian to stabilize in a state different from their initial Hamiltonian.

Close comparison of these conclusions with the conclusions arrived at in references [5,37], in the case of the stability of the hydrogen ground state orbital, reveals that the systems that they were referring to are the least action resonance volumes whose shapes and amplitudes can be determined by the wave function, each of which corresponding to one of the stable least action electromagnetic resonance states into which electrons become captive in atoms.

The related conclusion, drawn [5], is that the wave function describes the shape of the volumes occupied by the statistical spread of the positions that an electron can possibly occupy in the various orbital configurations as a function of local circumstances, as defined originally, while the resonance mechanics previously described explains the existence of these volumes and their elaboration as a function of time, as localized electrons are forced into constant resonance axial motion in reaction to the local magnetic interaction fluctuations; their permanent localization during the resonance process being established by correlating their point-like physical presence in space with the pointlike trispatial junction located in their center in the trispatial geometry.

Consequently, it can be observed that the Hamiltonian as currently defined deals at the general level with how the volume occupied by the statistical spread of one state can be made to evolve into one of the other authorized volumes, but in no way deals with the continued presence of the translationally hindered adiabatically induced unidirectional half of the electron carrying kinetic energy, which is now mostly acting axially toward the nucleus, while captive on a clearly definable axial resonance trajectory about a mean distance from the nucleus, while alternating between diminished and increased adiabatic induced energy intensity sequences, as the electron is forcibly pushed away from and then released to move back toward the nucleus within the volumes determined by the wave function [5].

\section{Repairing the submicroscopic momentum disconnect}

It is clear from the analysis carried out in references $[5,43]$ that the transversally oscillating electromagnetic half of the induced adiabatic carrying energy of charged elementary particles, that provides to the particle its omnidirectionally inert related mass increment, is not affected whether or not its unidirectional other half is prevented from being expressed as a translational velocity of the particle, while it is stabilized into one of the possible orbital resonance states in atoms.

On its part, the natural motion of the unidirectional half of the induced energy can be resisted translationally by local electromagnetic equilibrium states in a way that can only lead to the hindered velocity being expressed as a replacing "pressure" constantly exerted in the direction of the nucleus, given the opposite signs of the charges of the electron and that of the sum of the charges of the nuclei's nucleon internal charged components, which determine the vectorial direction of application of this pressure.

The predominantly repulsive magnetic interaction that counters the motion of the electron toward the nucleus can logically only be by nature a "contact" resistance between the spherically oscillating kinetic energy magnetic spheres of the particles involved, and of their carrying energy, "bumping" against each other, so to speak, within magnetostatic Z-space [12], which provides an elastic contact surface that opposes by structure, to the electron, the same type of hindrance to moving closer to the center of mass of the atom that the surface of the Earth opposes to bodies lying on the ground to moving closer to the center of mass of the Earth.

From the strict electromagnetic perspective, it must always be kept in mind that all macroscopic bodies lying on the ground, as well as all of the matter of which the ground is made at the surface of the Earth, are ultimately made up of atoms, whose ultimate building blocks are only electrons, up quarks and down quarks, which are the only stable scatterable point-like behaving, electrically charged and massive electromagnetic elementary particles that ever were detected inside atomic and nuclear structures by means of non-destructive scattering, and that are the only components of matter that can be induced with kinetic energy by the Coulomb force.

The charged particles making up these bodies lying at the surface of the Earth are consequently also in constant Coulomb force inverse square interaction function of distance with the charged particles making up the mass of the Earth, which consequently find themselves in the same situation as an electron being attracted to a proton by the Coulomb force in a hydrogen atom, even while being captive of each other in various least action electromagnetic equilibrium states to form these macroscopic masses [5].

In other words, this "pressure", now replacing the electron's inhibited velocity, in the direction of application of the unidirectional energy of its carrier-photon toward the proton, amounts to a "gravitational force" in newtons that the electron is applying toward the nucleus while captive at mean ground state orbital distance from the hydrogen atom.

In this regard, reference [4] clearly establishes the mutual identity of all classical force Equations by mathematically demonstrating that they all can be converted to $\mathrm{F}=\mathrm{ma}$, which includes the establishment of the identity between the macroscopic gravitational force with the Coulomb force, after having clarified that the gravitational constant that must be used in any natural axially structured many-bodies system must take into account the orbital parameters specific to the relative order of magnitude of that system for it to remain coherent with observed reality, whence the establishment of a gravitational constant specific to the hydrogen atom, ref: ([4], Equation (13)) reproduced here for convenience:

$$
\mathrm{G}_{\mathrm{p}}=\frac{4 \pi^{2} \mathrm{r}_{\mathrm{o}}{ }^{3}}{\mathrm{M}_{\mathrm{p}} \mathrm{T}^{2}}=1.514172983 \mathrm{E} 29 \mathrm{~N} \cdot \mathrm{m}^{2} / \mathrm{kg}^{2}
$$

where $\mathrm{M}_{\mathrm{p}}=1.67262158 \mathrm{E}-27 \mathrm{~kg}$ is the mass of the proton, $\mathrm{r}_{\mathrm{o}}=5.291772083 \mathrm{E}-11 \mathrm{~m}$ is the mean hydrogen ground state orbital radius, and $\mathrm{T}=1.519829851 \mathrm{E}-16 \mathrm{~s}$ is the time that would be required for the electron to orbit the proton one time at distance $\left(r_{o}\right)$ if it could so translate; in replacement of $(M)$, the mass of the Sun, $(r)$, the mean distance between the Earth and the Sun, and (T), the time taken for one orbit of the Earth about the Sun, which are the values embedded into the standard definition of astronomical constant G [4].

What allows using the potential time that the electron would take to travel once about the proton at distance $\left(r_{\mathrm{o}}\right)$ from the proton, as theoretically proposed in the Bohr atom, is the fact that the correct level of energy that would allow the electron to really move at the corresponding velocity is permanently induced by the Coulomb force at this distance of the nucleus of the hydrogen atom. So this 
time element is coherent with the quantity of motion of the fully expressed corresponding momentum even with its current definition, and can be calculated from the frequency of the carrying energy adiabatically induced at the mean Bohr radius (4.359743808E-18 j), which corresponds to the number of times the electron would orbit the nucleus at distance $\left(r_{0}\right)$ in 1 second at the corresponding velocity:

$$
\mathrm{T}=1 \mathrm{sec} / 6.57968391 \mathrm{E} 15 \mathrm{~Hz}=1.519829851 \mathrm{E}-16 \mathrm{sec} .
$$

This velocity replacing "pressure" now oriented toward the nucleus corresponds to the well known "force" of $8.238721759 \mathrm{E}-8$ newtons applicable to the mean hydrogen ground state orbital, and is put in correct perspective as calculated ([4] Equation (14)), reproduced here for convenience:

$$
\mathrm{F}_{\mathrm{g}}=\frac{\mathrm{e}^{2}}{4 \pi \varepsilon_{\mathrm{o}} \mathrm{r}_{\mathrm{o}}{ }^{2}}=\mathrm{G}_{\mathrm{p}} \frac{\mathrm{M}_{\mathrm{p}} \mathrm{m}_{\mathrm{e}}}{\mathrm{r}_{\mathrm{o}}{ }^{2}}=8.238721759 \mathrm{E}-8 \mathrm{~N}
$$

\section{Conclusion}

Observing that physical reality was of necessity explored from our macroscopic perceptions digging inwards toward the submicroscopic level as more and more understanding was gained about the nature of matter, mass and energy, which eventually led to important issues remaining unresolved despite our current rather deep knowledge base, it appeared interesting to attempt addressing these issues from what was now known about the submicroscopic level, building upwards toward our macroscopic level.

Analysis of this knowledge base then allowed identifying the electromagnetic properties of energy as ruling this ultimate bottom of the submicroscopic level of physical reality, where only one energy inducing force can be identified, which is the Coulomb force as previously put in perspective.

This perspective also brings to light two major aspects of electromagnetic elementary particles that turn out not to have yet been taken account of in the currently useful theories that were developed over time, which is the fact that the current mechanics theories do not take into account the physical presence of the elementary charged and massive particles of which they are made and of the consequences of their individual motion on the state of motion of the macroscopic bodies to which they belong, as exemplified by the issue that this situation raises with respect to macroscopic rotating bodies for example, and the fact that quantum mechanics and electromagnetism do not yet integrate the mandatory internal mutual induction of the electric and magnetic aspects of electromagnetic energy quanta in a manner that mechanically explains why these quanta can be self-sustaining in a localized manner and behave point-like during scattering encounters.

Interestingly, this proposed alternate foundation of physical reality seems to directly correlate with the zero-point energy level of the quantum vacuum concept that postulates a hypothetical uniform zero point energy excitation level of the quantum vacuum at the beginning of the universe, which is the foundation QFT. The main difference is that this alternate foundation proposes a hypothetical uniform zero energy level in space at the beginning of the universe, that then provides a continuous infinitesimally progressive interaction alternative that offers seamlessly workable mechanical solutions that QFT does not provide, which are, among other benefits, a Maxwell Equations compliant mechanical description of the internal self-sustaining mutual induction of the electric and magnetic fields of the localized energy quantum constituting each electromagnetic photon [12] and of the invariant rest mass of each charged and massive elementary particle
[35], clear separation of the also electromagnetic carrying energy of elementary particles from the energy making up their invariant rest mass $[14,32]$, which allows becoming aware of the adiabatic nature of this carrying energy induced in all charged elementary particles as a function of the distances separating them [5], and an electromagnetism compliant mechanical explanation of the stability of electronic as well as nucleonic resonance orbitals states [5,37].

Considering that at the beginning of the universe, the ultimate bottom of the submicroscopic level would have been an energyless static empty vacuum devoid of any charged particles that the Coulomb force could have caused to interact, instead of the quantum vacuum zero energy point proposed by QFT that creates particlesantiparticles pairs by means of assumed spontaneous natural quantum vacuum fluctuations, obviously raises the question of how the first electromagnetic photons could have appeared at the origin of the universe, when no charged particles even existed to be accelerated to eventually liberate the first bremmsstrahlung photons that are required from this perspective to mutually destabilize in a process whose existence was confirmed by K. McDonald et al. in 1997 at the SLAC facility [44] into producing the first ever electron-positron pairs that could then be accelerated by this inducing force and be induced with the first ever adiabatic carrying energy quanta, eventually leading to the production of the first nucleons and first hydrogen atoms.

This issue, that of course remains pending, is analyzed [45] where it is tentatively addressed by the idea that the constancy of the flow of time may also be kinetic energy driven and that some punctual event in the far past could have momentarily impeded its motion, thus triggering the release in space of the initial electromagnetic quanta as energetic bremmsstrahlung photons, thus initiating a charged particles generation process that would still be ongoing $[33,41]$.

The concept of electromagnetic elementary particles self-energy of QFT is replaced by the mechanically definable concept of selfsustaining mutual induction of elementary particles' electric and magnetic aspects of the energy of the localized quanta of elementary charged particles $[12,13,35]$.

The underlying force being statically present and in permanent action between each pair of charged particles, each occurrence of such interaction between charged pairs can then be seen as one unit occurrence among the multitude of such occurrences constituting a universal gradient made strictly of the addition of all such active occurrences between all existing charge pairs in the universe. Contrary to QFT, where the presence of individual excited states affects the intensity of the local energy gradient, the presence of two electromagnetic particles is required for each discrete unit Coulomb force interaction occurrence to exist in the universal gradient, so the gradient is one of intensity of these interaction occurrences and not directly one of energy intensities, or density, as in QFT.

Although the gradient involves the Coulomb force, it does not involve the traditional continuous electric field associated with this force, but uniquely the limited set of all really existing discrete interaction occurrences at play between the really existing charges in the universe as a discontinuous assembly of individual occurrences.

It becomes possible now to separate this gradient into four ranges of intensity levels, whose limits correspond to the various resonance intensity ranges that can be identified in nature. As put in perspective [33], the most intense level is determined by the resonance states characterizing charged elementary particles interactions within nucleons. The second level applies to nucleons stabilization within 
nuclei. The third level applies to electronic resonance states within atoms and molecules, as well as between atoms and molecules in direct contact with each other in any local accumulations of matter. And finally, a forth and ultimate level of intensity applies to all atoms, molecules and larger bodies in state of freefall, a category that includes macroscopic orbits stabilization at the astronomical level.

These various ranges of intensity of induction of adiabatic carrying energy by the Coulomb force, one of whose major component is the permanently induced adiabatic mass increment that it provides for each existing charged particle, can then be directly related to the 4 forces of the Standard model as put in perspective [33], four forces which then turn out to only be approximate alternate representations of the various intensity ranges of application of the same underlying Coulomb adiabatic energy inducing force.

It is consequently at this point that a clear relationship can be established between quantum mechanics and this global gravitational gradient since the wave function establishes with precision the locations and shapes of the volumes within which each electron stabilizes into its least action electromagnetic resonance equilibrium orbital states by means of one interaction occurrence of the gradient, as clarified [5] and is consequently related to the third intensity range of the universal interactions intensity gradient. This interaction occurrence can then be recognized as a one local occurrence of the classical "gravitational force" acting as a function of the inverse square of the distance between the electron and each of the charged elementary subcomponents of the nucleus, each of them corresponding to an occurrence of the tertiary attractors category described [33].

Each element of the global gradient contributes to the distance coupled adiabatic energy induction variations imposed on the charged particles by the local dynamic circumstances that define their local effective masses. That is, dynamic circumstances that evolve over time according to the rate of matter accumulation in stellar bodies, one of the most remarkable process of which, is the mechanical process that relates the stars ignition threshold to the progressive adiabatic compression of the hydrogen atoms ground state orbitals as depth increases toward the center of protostar masses, due to accumulation of primordial hydrogen atoms, up to the point at which their ground state orbital reaches the axial distance within hydrogen atoms at the center of such masses, that provides them with the energy level that triggers the neutron nucleogenesis process that initiates the fusion process, as also analyzed [33].

Interestingly, given that up and down quarks resonance states inside nucleons are by structure submitted to the same electromagnetic resonance mechanics as electrons in atomic orbitals, it can be concluded that the various wave function representations of quantum mechanics could be adapted to directly apply to them within nucleons in a much more integrated and satisfactory way than QCD allows, which would associate quantum mechanics to the most intense intensity level of the gravitational gradient.

Finally, given the variability function of distance of the size of the adiabatic mass increments which are part of any amount of carrying energy induced in charged elementary particles by the Coulomb force, represented in Equations (10) and (14) as determined from the analyses carried out in references [14,32], it can be observed that the sum of the experimentally confirmed maximum invariant masses of the three up and down quarks constituting the interacting inner structure of protons and neutrons, amounts to barely from $2 \%$ to $2.4 \%$ of the measured masses of these nucleons, and that consequently more than $97 \%$ of the masses of all massive bodies in existence can only be of adiabatic origin and are thus part of the carrier-photons of charged and massive elementary electromagnetic particles $[5,13]$.

This means that the mass of nucleons can vary as a function of the local intensity of the gravitational gradient and that more than $97 \%$ of the measurable mass in the universe is adiabatically induced by the Coulomb force in this manner, which reveals that the mass of astronomical bodies is also variable as a function of the distances separating them [33].

\section{References}

1. Michaud A (2017) Electromagnetic mechanics of elementary particles. (2nd edn). Scholar's Press. Saarbrücken Germany 2016

2. Resnick R, Halliday D (1967) Physics. John Wyley \& Sons, New York, USA.

3. Sears F, Zemansky M, Young $H$ (1984) University physics. (6th edn), Addison Wesley, USA.

4. Michaud A (2013) Unifying all classical force equations. Int J Eng Res Dev (IJERD) 6: 6, 27-34.

5. Michaud A (2016) On adiabatic processes at the elementary particle level. J Phys Math 7: 177 .

6. Kaufmann W (1903) Über die "Elektromagnetische Masse" der Elektronen Kg Gesellschaft der Wissenschaften Nachrichten Mathem -Phys Klasse 91-103.

7. Cauchois Y (1952) Atomes Spectres Matière Éditions Albin Michel, Paris.

8. Abraham M (1902) Dynamik des Electrons Nachrichten von der Gesellschaft der Wissenschaften zu Göttingen Mathematisch-Physikalische Klasse S 20.

9. Ernst A, Hsu JP (2001) First proposal of the universal speed of light by Voigt in 1887. Chinese Journal of Physics 39: 3.

10. Poincaré H (1905) La valeur de la science France Flammarion, 1994 Edition.

11. Haskell RE (2003) Special relativity and Maxwell's equations. Computer Science and Engineering Department, Oakland University Rochester, MI 48309, USA.

12. Michaud A (2016) On De Broglie's double-particle photon hypothesis. J Phys Math 7: 153.

13. Michaud A (2013) The mechanics of neutron and proton creation in the 3-spaces model. Int J Eng Res Dev (IJERD) 7: 9 29-53.

14. Michaud A (2013) From classical to relativistic mechanics via Maxwell. Int J Eng Res Dev (IJERD) 6: 4 1-10.

15. Marmet $P$ (2003) Fundamental nature of relativistic mass and magnetic fields International IFNA-ANS Journal No 3 Vol 9 Kazan State University Russia.

16. Ciufolini I, Wheeler JA (1995) Gravitation and inertia. Princeton University Press, USA

17. Feynman RP, Leighton RB, Sands M (1964) The Feynman lectures on physics. Addison-Wesley, II: 28-1.

18. Breidenbach M, Friedman JI, Kendall HW (1969) Observed behavior of highly inelastic electron-proton scattering. Phys Rev Let 23: 16 935-939.

19. Blackett PMS, Occhialini G (1933) Some photographs of the tracks of penetrating radiation. Proceedings of the Royal Society 139: 699-724.

20. Anderson JD, Laing PA, Lau EL, Liu AS, Nieto MM, et al. (2005) Study of the anomalous acceleration of Pioneers 10 and 11 .

21. Keith JC (1963) Gravitational radiation and aberrated cenripetal force reactions in relativity theory Part 2 retarded cohesive forces. Revista Mexicana de Fisica XII: 1

22. Fremerey JK (1973) Significant deviation of rotational decay from theory at a reliability in the $10^{-12} \mathrm{sec}^{-1}$ range. Phys Rev Lett 30: 16 753-757.

23. Blewett JP (1946) Radiation losses in the induction electron accelerator. Phys Rev 69: 87.

24. Einstein A, Schrödinger E, Pauli W, Rosenfeld L, Born M, et al. (1953) Louis de Broglie physicien et penseur. A tribute to Louis de Broglie for his $60^{\text {th }}$ birthday, each colleague providing one chapter. Einstein even collaborating to 2 distinct chapters, the complete text drafting a detailed overview of the state of knowledge in fundamental physics in 1952. Éditions Albin Michel, Paris. 
Citation: Michaud A (2017) Gravitation, Quantum Mechanics and the Least Action Electromagnetic Equilibrium States. J Astrophys Aerospace Technol 5: 152. doi:10.4172/2329-6542.1000152

25. De Broglie L (1934) L'équation d'ondes du photon C R Acad Sci 199: 445-448.

26. De Broglie L, Winter MJ (1934) Sur le spin du photon C R Acad Sci 199: 813-816.

27. De Broglie $L$ (1936) La théorie du photon et la mécanique ondulatoire relativiste des systèmes C R Acad Sci 203: 473-477.

28. De Broglie $L$ (1937) La quantification des champs en théorie du photon $C R$ Acad Sci 205: 345-349.

29. De Broglie L (1993) La physique nouvelle et les quanta Flammarion France 1937 (2nd edn) 1993 with new 1973 Preface by Louis de Broglie.

30. Michaud A (2000) On an expanded Maxwellian geometry of space. Proceedings of Congress-2000 - Fundamental Problems of Natural Sciences and Engineering 1: 291-310.

31. Michaud A (2017) The last challenge of modern physics. J Phys Math 8: 217.

32. Michaud A (2007) Field equations for localized individual photons and relativistic field equations for localized moving massive particles. International IFNA-ANS Journal No. 2 13: 123-140 Kazan State University, Kazan, Russia.

33. Michaud A (2013) Inside planets and stars masses. Int J Eng Res Dev (IJERD). 8: 1 10-33.

34. Michaud A (2013) The expanded Maxwellian space geometry and the photon fundamental LC equation. Int J Eng Res Dev (IJERD) 6: 8 PP 31-45.

35. Michaud A (2013) The mechanics of electron-positron pair creation in the 3-spaces model. Int J Eng Res Dev (IJERD) 6: 10 01-10.

36. Lowrie W (2007) Fundamentals of geophysics. (2nd edn), Cambridge University Press, USA.

37. Michaud A (2013) On the magnetostatic inverse cube law and magnetic monopoles. Int J Eng Res Dev (IJERD) 7: 5 50-66.

38. Kotler S, Akerman N, Navon N, Glickman Y, Ozeri R (2014) Measurement of the magnetic interaction between two bound electrons of two separate ions Nature magazine. 510: 376-380.

39. Golovko VA (2008) Electromagnetic radiation and resonance phenomena in quantum mechanics.

40. Soosaleon A, Ambrose S (2017) Gravity induced resonant emission.

41. Michaud A (2013) The corona effect. Int J Eng Res Dev (IJERD) 7: 1101-1109.

42. Born M, Fock S (1928) Beweis des Adiabatensatzes Zeitschrift für Physik. 51: 165180.

43. Michaud A (2013) On the Einstein-de Haas and Barnett Effects Int J Eng Res Dev (IJERD). 6: 1207-1211.

44. McDonald K, Smith GH (1997) Positron production in multiphoton light-by-light scattering. Phys Rev Lett 79: 1626.

45. Michaud A (2016) The birth of the universe and the time dimension in the 3-spaces model. Am J Phys Special Issue: Insufficiency of big bang cosmology 5: 44-52. 